\title{
Human endocrine system modeling based on ontologies
}

\author{
Pamela Viale ${ }^{\mathrm{a}, \mathrm{c}, *}$, Juan José Bora ${ }^{\mathrm{a}}$, Maximiliano Benegui ${ }^{\mathrm{a}}$, Marta Basualdo ${ }^{\mathrm{a}, \mathrm{b}}$ \\ a UNR - FCEIA, Facultad de Ciencias Exactas, Ingeniería y Agrimensura, Universidad Nacional de Rosario, Rosario, Argentina \\ ${ }^{\mathrm{b}}$ UTN - FRRO, Facultad Regional Rosario, Universidad Tecnológica Nacional, Rosario, Argentina \\ ' UCA, Facultad de Química e Ingeniería "Fray Rogelio Bacon", Pontificia Universidad Católica Argentina, Campus Rosario, Argentina
}

\section{A R T I C L E I N F O}

\section{Article history:}

Received 29 December 2015

Revised 2 August 2016

Accepted 5 August 2016

Available online $\mathrm{xxx}$

\section{Keywords:}

Ontology

Knowledge engineering

Knowledge base

Diabetes mellitus

E-Health

\begin{abstract}
A B S T R A C T
This article presents a novel use of an ontological approach of a rigorous generic model of the human endocrine system. It is based on an existing ontology specifically developed for chemical engineering design, named OntoCAPE. It provides most of the necessary concepts for implementing compartmental models of the human endocrine system, such as the UVa/Padova model, ${ }^{1}$ accepted by the FDA. ${ }^{2}$ We named this extended ontology Bio OntoCape which is connected with MatLab to perform dynamic simulation with the constructed model to predict the impact of the external stimuli such as meals intake and insulin dosage. This mathematical model was chosen because is enough versatile to represent healthy, prediabetic and diabetic persons. The complete system is thought to be helpful for participants from different disciplines, such as, endocrinologists, nutritionists, nurses, engineers and patients among others. In addition, it is envisioned that this development can be extended to configure an e-Health platform for diabetic patients treatment in Argentina. This will help to remotely monitoring patients reducing the personal attendance at hospitals as well as medical budgets.
\end{abstract}

(c) 2016 Elsevier B.V. All rights reserved.

\section{Introduction}

This work presents a first attempt to implement knowledge related to bioengineering study that centers the attention on diabetic patients treatment in the context of ontologies. Since an ontology is considered as "an explicit specification of a conceptualization" [9], it allows to represent consensual knowledge about a specific domain. Hence, ontologies represent a valuable tool for the ambitious project of constructing an e-Health platform for diabetes care. The focus of this article is to present an ontology-based modeling of the human endocrine system taking into account bioengineering elements.

For modeling the human endocrine system, we chose an existing ontology called OntoCAPE [16,18]. This decision was based taking into account the recommendations of the work of Bogle et al. [10] who emphasizes the potential role for Computer Aided Process Engineering (CAPE) in developing engineering analysis and design

\footnotetext{
* Corresponding author.

E-mail addresses: pamela@fceia.unr.edu.ar, pameviale@gmail.com (P. Viale), borajuanjo@gmail.com (J.J. Bora), maxibenegui@yahoo.com.ar (M. Benegui), mbasualdo@frro.utn.edu.ar, basualdo@fceia.unr.edu.ar (M. Basualdo).

1 The UVa/Padova Type 1 Diabetes Mellitus Metabolic Simulator is a computer model of the human metabolic system based on the glucose-insulin dynamics in human subjects

2 U.S. Food and Drug Administration
}

approaches to biological systems. The similarity between modeling chemical process and bio process and the availability of devices models such as sensors, valves, pumps, etc. and control algorithms too, were the main reasons for choosing OntoCAPE. It has been originally developed to support and simplify the development of software applications in CAPE, therefore, it is a good candidate to be extended to bio systems. We propose a customized knowledgebase from OntoCAPE, which contains the knowledge considered relevant for modeling compartmental models of the human endocrine system. We named this extension Bio OntoCape.

In the past few years, scientific community, mostly coming from medical domain, has involved many efforts and resources in the development of semantic technology. Legaz-García et al. [14] proposed semantic interoperability for using the valuable information of electronic health care records (EHR) data. Many web services platforms have been developed with the aim of assisting users with health information. One of these platforms is the Personalized Information Platform for Health and Life Services (PIPS) [2]. It combines a number of technologies in order to give advices to users. It is based on an ontological approach to achieve a common understanding of the domains in which the system operates. At work [1] a framework for diabetes education content management in accordance to semantic web concepts is described. The world wide web offers a wealth of information resources on diabetes mellitus that can answer most of the knowledge needs of clinicians and their patients. The aim of the project was to make 
medical web resources more meaningful to computers to reduce the risk of overloading patients and medical practitioners with unnecessary or low quality information. At work [19] is proposed to integrate multidimensional data warehousing with data mining tools and ontologies. This is because ontologically described multidimensional data warehouse and mining facilitates the knowledge extraction. Rajbh et al. [20] describes a Medical Information System based on semantic web technology for sharing data between different hospitals. The heterogeneous nature of the health care data makes it a very suitable candidate for Semantic Web Application. The knowledge-based telemonitoring platform described in [23] is another example of a remote health-care application. This healthmonitoring system checks a patient health status using a smartphone. The knowledge-base design involved ontologies and various reasoning methods. All of these cited works constitute examples of the development of semantic web technologies for health care.

The modeling of knowledge using ontologies enables the interoperability needed by the e-Health platform for the assistance of diabetic patients. This platform would be used by different actors like doctors, nurses, endocrinologists, chemical engineers, computer science specialists and, of course, also the patients. The ontology will allow to overcome the problem associated to the usage of different terminology handled by the different actors that will use the platform. Search engines can then work on a particular concept rather than trying to find ambiguous keywords. In the particular case of the e-Health platform, this feature is very advantageous for a patient who wishes to inquire about his treatment. This would increase the involvement of the patient into his treatment that would impact in the quality of his life.

The platform aims to reduce the number of visits to the doctor. This would be a great benefit for patients with low resources as well as those patients with difficulties to follow any treatment. All ontologies that will be developed within the project must be created having in mind that they must be coupled together to improve the quantity and quality of knowledge about the treatment of a particular person or a group of people. To resume, ontologies provide a suitable inter-semantic framework for a diabetic patient through which he may have a better control over his treatment, his blood glucose levels and a better understanding of his body behavior, which will help to achieve a better quality of life.

\section{Motivation}

Diabetes mellitus is a chronic disease, which in recent years has become an epidemic. This disease shows up when the pancreas can not produce enough insulin (Diabetes mellitus type I - DMTI) or when the produced insulin does not have the necessary properties to be effective for a person's body (Diabetes mellitus type II - DMTII). The insulin is a hormone that allows to metabolize the glucose from ingested food for being then used by the body cells to produce the necessary energy for muscles and tissues.

According to the last World Health Organization (WHO) study of global burden of disease, diabetes is the 19th leading cause of disease burden, and it is expected to rise to the 10th position by the year 2030. In Latin America, the situation is also discouraging, diabetes is the 6th cause of disease burden. In Argentina, diabetes affects $9.6 \%$ of the adult population, being DMTII the most common (90\%) clinical form [8].

Diabetes is an expensive disease in Argentina as well as worldwide and its prevalence is continuously rising, affecting the quality of life of people and their life expectancy. It also imposes a heavy burden to the argentinean health care budget and on the economy in the form of productivity losses [3]. Overall diabetes prevalence increased from $8.4 \%$ in 2005 to $9.6 \%$ in 2009 at national level. In 2009 , diabetes was the 7 th leading cause of death with a mortal- ity rate of 19.2 per 100,000 habitants. The per capita hospitalization cost for people with diabetes was significantly higher than for people without the disease, US\$ 1,628.- vs. US\$ 833.- in 2004.

Mathematical modeling is now widely applied in physiology and medicine to support the life scientist and clinical worker . Our aim in writing this article is to provide an introduction to this topic, presenting the underlying principles of good modeling methodology together with specific example of the endocrine system based on the expert knowledge given at the book [4] which is very useful for diabetic patients. Mathematical modeling finds application in medical research, in education and in supporting clinical practice. In the research context, the use of models can, for example, yield quantitative insights into the manner in which physiological systems are controlled. In the educational setting, medical students can use computer model simulation to explore the dynamic effects of pathophysiological processes or of drug therapy. In the clinical area, mathematical models can enable estimates to be made of physiological parameters that are not directly measurable enabling predictions to be made as to how changes in drug therapy will impact on variables of clinical importance such as blood glucose concentration. This article could be of interest to a wide range of student and practitioner backgrounds. In terms of the student readership, it is designed to appeal to biomedical engineers and to others studying physical and engineering sciences, and biological and life sciences. It should also appeal to medical students who wish to enhance their quantitative understanding of the physical and chemical processes that shore up physiology and medicine. Further, this article should be of interest to practitioners of bioengineering who have an interest in quantitative aspects of physiology and medicine. This article is based on the exploration of some of the complexities of physiology involved in the human endocrine system and their quantitative features where the ontologies could help to be better understood. The concepts used here for constructing efficient mathematical models with the help of ontologies could be considered as a novel way to be used for a wide range of purposes: to gain insights, to support processes of measurement, to make predictions of future behavior and in a variety of ways assist in enhancing clinical research and practice. A number of approaches to developing mathematical models mainly based on first principles concepts are then considered, with each being illustrated by three examples. Ensuring that a mathematical model is valid; that is to say fit for its intended purpose. Hence, the objective here is to demonstrate, how to handle the modeling concepts, methods and techniques that are described and discussed here can be extended to other real-world problems in physiology and medicine. However, since the physiological modeling is very much an interdisciplinary subject, the topic is also central to a range of related disciplines including biomathematics, biochemical, medical and health informatics and systems physiology. The focus of this present article is the use of ontology to provide a comprehensive introduction to the modeling of dynamic, physiological endocrine systems. The emphasis is placed firmly on developing sound modeling methodology, with some examples included as illustrations. Then, to achieve our purpose we had into account that the most recently, ontological software engineering has developed into a scientific field of its own, which puts particular emphasis on the theoretical foundations of representation and reasoning, and on the methods and tools required for building ontologybased software applications in diverse domains. Though this field is largely dominated by computer science, we propose an interdisciplinary work to achieve close relationships to be established with its diverse areas of application. Hence, the researchers involved in this area are interested in exploiting the results of ontological software engineering, particularly to build large knowledge-intensive applications at high productivity and low maintenance effort firstly dedicated to the problem of diabetes care. We detected very 


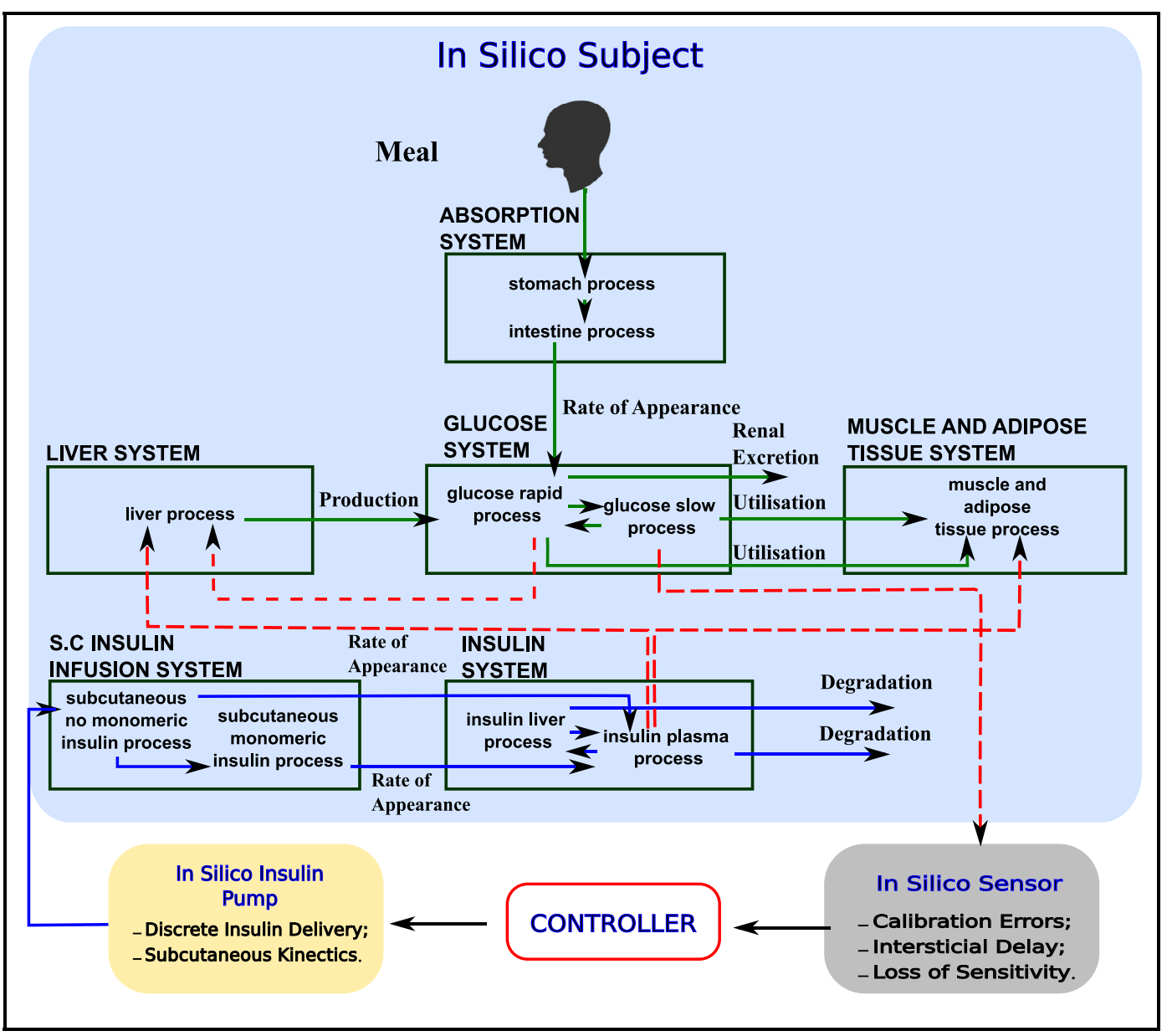

Fig. 1. Scheme of the UVA/Padova glucose-insulin system.

little activity in the field of engineering sciences. In particular, very few ontologies have been elaborated for the domain of biochemical engineering. In this context, as starting point we looked for an existent ontology that at least be able to provide a reasonable conceptualization of the chemical engineering domain, which is a prerequisite for establishing a shared understanding of concepts and terms in a certain scientific field and for fostering the communication in a typical cross-disciplinary engineering design team. We found the ontology named OntoCape which is able to support and to simplify the development of future software applications in CAPE, a sub-discipline of chemical and biochemical engineering. Besides this very concrete engineering objective, the development of OntoCAPE also aimed at the elucidation and the benchmarking of architectural principles for the design of largescale ontologies, which can be reused in the same domain for different applications or even across related (engineering) domains. Therefore, OntoCape was considered the best possible candidate to develop a novel ontological software for biochemical engineering field able to capture the main conceptualization for constructing physiological models.We distinguish this extended concepts under the new name: Bio OntoCape.

Some strategies have already been implemented to decrease the associated costs of diabetes care for the argentinean health system. The Program for the Prevention, Care and Treatment of People with Diabetes (PROPAT) has been applied to the social security sector from 1998 to 2000. This program led to significant improvement in clinical and biochemical indicators and higher quality of care for people with DMTII. This was observed also in the reduction of the number and duration of hospitalizations of diabetic patients.
The development of a diabetic patients knowledge base could be relevant for helping educational programs like PROPAT. This would facilitate the manipulation of information of diabetic patients, improving their education for achieving better self-control techniques and reducing their dependence of experts.

\section{Mathematical model of the human endocrine system}

In the area of diabetes, accurate prediction of clinical trials has been done by the Archimedes diabetes model [6,7]; a company - Entelos, Inc. - specializes in predictive biosimulation developed a complex model for diabetes simulations. These existing diabetes models, however, are based on population models. Hence, their capabilities are limited to prediction of population averages that would be observed during clinical trials. In this work, we focus on capturing the main concepts for constructing the mathematical model of the human endocrine system. The methodology is very similar to that used for chemical process modeling. The final purpose is that the model be implemented for dynamic simulation of a patient to prevent undesirable dangerous situations for the health of the patients. All the necessary information and data were presented in the patent [11]. This model was chosen because of its novelty and strength since it is based on virtually model-independent measurements of the various glucose and insulin fluxes occurring during a meal which represent the main information for diabetes care. The endocrine system is very complex and only the availability of glucose and insulin fluxes, in addition to their plasma concentrations, have allowed to minimize structural uncertainties in modeling other processes. The 
model may comprise of 12 nonlinear differential equations, 18 algebraic equations and 35 parameters. The complete description of those elements are given at the Appendix A. As a result a user-friendly model, able to be implemented for simulation purposes is of great help for investigators of several disciplines without a specific expertise in modeling. This general model allows to study both normal and pathological conditions, e.g. type 2 diabetes (DMTII), prediabetic and open-and closed-loop insulin infusion in type 1 diabetes. The equations describe the relationship between plasma glucose and insulin concentrations, and glucose and insulin flows. The endocrine system is divided into different subsystems which are divided into compartments. The parameters are adjusted based on experiments over 300 normal subjects in order to obtain a non-diabetic model. The Glucose Insulin Model (GIM), is also known as the UVa/Padova simulator because it was developed by the University of Virginia, USA, and the University of Padova, Italy, adapts the previous non-diabetic model to be able to represent a DMTI subject. The extension to prediabetic and DMTII can be found at the patent description [13]. The endocrine system model includes a model of subcutaneous insulin kinetics, and a higher endogenous glucose production as shown in Fig. 1. The glucose flows are represented using green arrows, the insulin flows with blue arrows and the signals by dotted red arrows. The complete model equations included in GIM are given at the appendix.

Fig. 1 shows the main elements of the mathematical model which can be implemented for computer simulation environment acting as the in silico patient behavior working in a closed-loop control system in case the artificial pancreas is implemented in a DMTI patient. In the following explanation the list of elements are briefly detailed:

1. A sufficiently large cohort in silico subjects based on real individual data and spanning the observed variability of key parameters in the general population. In the proposed implementation [12] the simulated "cohort" includes $n=300$ simulated subjects in three age groups: 100 adults, 100 adolescents, and 100 children. At patent [11] data for 30 patients is available.

2. Sensor-specific simulator for emulating the possible sensor error capable of reproducing time lag, system and calibration bias, and random noise of subcutaneous CGM devices.

3. The model of insulin kinetics in the subcutaneous space tacking into account that insulin pumps typically deliver discrete insulin subcutaneously, one has to consider the inherent time lag for the insulin transport to the plasma.

4. In case of healthy person, prediabetic and DMTII the insulin is provided by the insulin subsystem without the pump model.

The data base available in patent [13] used to identify the model consisted of 35 subjects (prediabetes), and 23 DMTII patients who underwent a triple tracer meal protocol. Hence, it was possible to obtain in a virtually model-independent fashion the time course of all of the relevant glucose and insulin fluxes during specific meal. The authors reported subject characteristics such as average plasma glucose and insulin concentration, Ra, EGP, U and SR in prediabetes and DMTII together with the profile obtained in a matched healthy population. In all cases 26 parameters have to be given to complete the endocrine system model.

It must be noted that this work is focused on the main concepts related to generic endocrine system models construction through the use of ontologies but is also needed to create a comprehensive algorithm testing environment to evaluate the dynamic behavior of the main variables. In this context, we propose here to integrate our ontological representation with the simulation platform Simulink ${ }^{\circledR}$, which is part of the well known scientific soft- ware MATLAB ${ }^{\circledR}$ which will be described in this work. To do this implementation we had into account that simulation methods reported into a computerized simulation platform [12].

In the next section, the OntoCAPE ontology as well as the ontological representation of the human endocrine system will be introduced.

\section{Ontological approach}

\subsection{Introduction to ontologies}

Ontologies have the function of providing a consensual knowledge representation, which can be reused and shared across software systems and by different groups of users. To convert an ontology into a knowledge base for a particular application, the knowledge must be specialized and customized. Thus, we propose to extend and customize OntoCAPE ontology for building the knowledge base needed by the e-Health platform envisioned.

\subsection{OntocapE general description}

OntoCAPE is a fully elaborated formal ontology for the domain of chemical engineering $[16,18]$. As its names lets understand, OntoCAPE has been developed to linking ontologies with CAPE, as well as to facilitate the derivation of customized knowledge bases.

Considering the usability and reusability problems, OntoCAPE has been subdivided into different levels of abstractions. This strategy allows to separate general knowledge from knowledge about specific domains and applications. Besides, OntoCAPE modularization has been realized by partitioning the ontology into modules and partial models and a user can choose to reuse only a selected part of the ontology. OntoCAPE is formally specified using the Ontology Web Language OWL, in particular, OWL-DL. The entire ontology consists of 62 OWL files, each describing one module of the ontology. The following paragraphs describe the general structure of OntoCAPE (a more detailed description is available in [16]). An overview of OntoCAPE layers and principal modules is shown in Fig. 2.

The Meta Layer is the highest layer of OntoCAPE which has two functions: firstly, it explicitly represents OntoCAPE design principles and, secondly, it establishes common standards for the design and organization of the ontology. So, this Meta Layer supports ontology engineering and it ensures a consistent modeling style providing a guide for the extension and/or modification of the ontology. Specifically, this layer introduces key terms independently of any domain and establishes a mereotopological theory. Furthermore, generic design patterns are introduced, which define solutions and best practices for general design problems for lower level layers.

The Upper Layer of OntoCAPE introduces key concepts, which are specialized and refined in lower layers.

The Conceptual Layer of OntoCAPE is represented by four main partial models. The central model is the chemical_process_system model and it represents all concepts that are directly related to materials processing and plant operations. The material partial model provides an brief description of the materials involved in chemical processes, while the mathematical_model model defines the concepts required for a description of mathematical models and modeling. Finally, the supporting_concepts partial model provides fundamental notions as space, time, physical dimensions, unit systems, mathematical relationships, etc; not belonging directly to the CAPE domain but that are required for the definition or as a supplement to the domain concepts.

The Application-Oriented Layer, extends the Conceptual Layer adding classes and relationships necessary for practical use of ontology. 


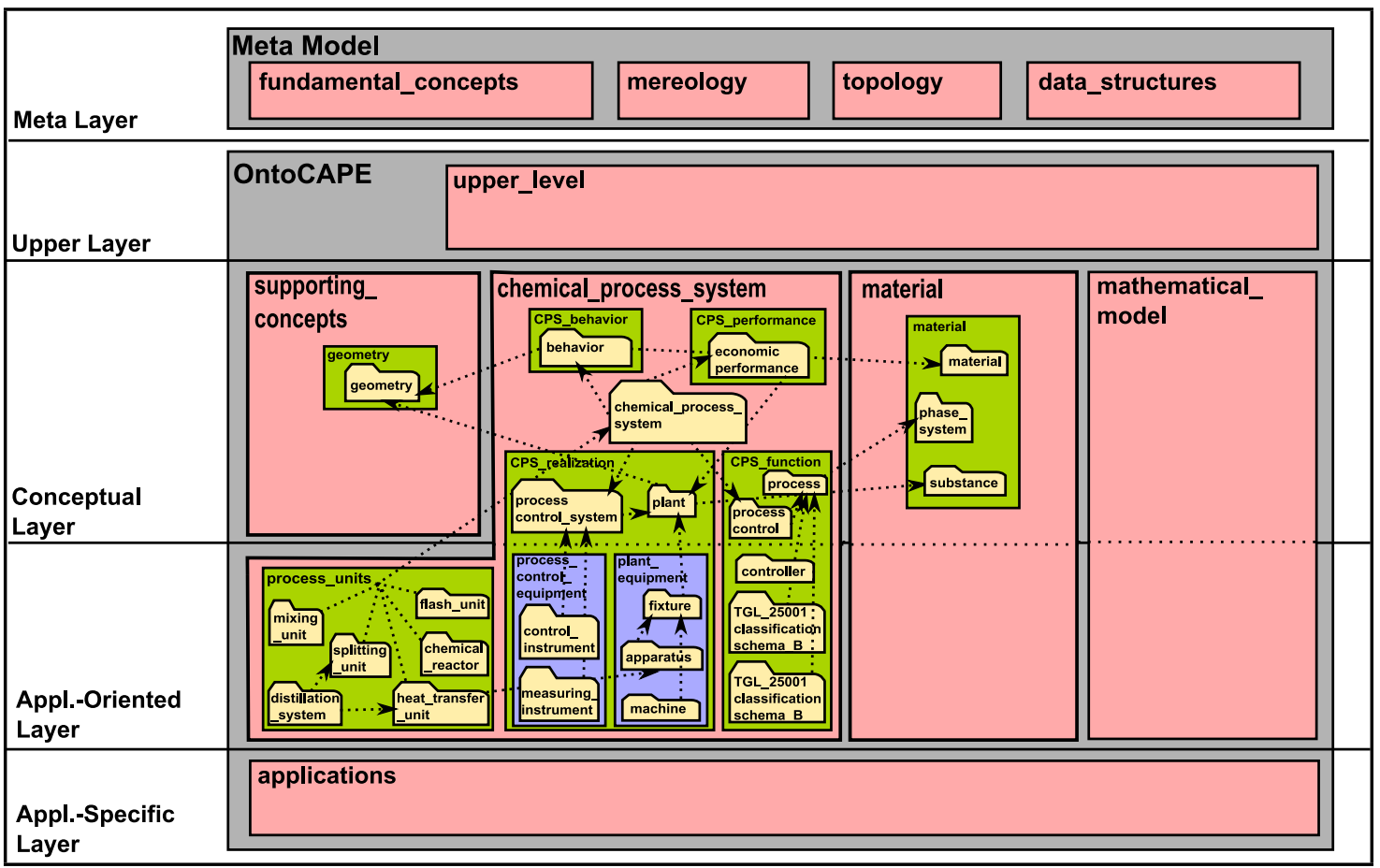

Fig. 2. Overview of OntoCAPE.

The Application-Specific Layer contains classes and relationships required for particular tasks and applications.

\subsection{Modeling the human endocrine system}

The human endocrine system is a biological chemical process system which can be interpreted as chemical plant. As OntoCAPE has been developed to help in the derivation of knowledge bases in the domain of chemical engineering, we choose this ontology as the starting point to represent the human endocrine system. Moreover, the attention has been centered on the chemical_process_system model which introduces most of the necessary definitions useful for representing human physiology aspects .

The chemical_process_system model is represented at Fig. 2. As can be seen it is composed by seven partial models that belong to the Conceptual Layer as well as the Application-Oriented Layer. The partial models that are part of the chemical_process_system model are: CPS_behavior, CPS_function, CPS_performance, CPS_realization, process_units, plant_equipment and process_control_equipment. Besides, chemical_process_system is able to import modules from other partial models.

The partial models CPS_behavior, CPS_function, CPS_performance, CPS_realization define the model in the Conceptual Layer. CPS_function describes the desired behavior of the chemical process system. CPS_realization represents the physical constitution of the process system, such as plants equipments and any technology present in the plant. CPS_behavior describes how the process system operates under certain conditions. Finally, the CPS_performance model is introduced to evaluate the performance of the system.

If we concentrate on the Application-Oriented Layer, we can see that the process_control_equipment and plant_equipment are specializations of the partial model CPS_realization. They give more detail information about machines or instrumentation in the plants.

Finally, the partial model process_units are examples of typical equipment of chemical_process_systems.
4.4. Bio ontocape development for modeling the human endocrine system

In this section we describe how we extended OntoCAPE to represent the human endocrine system in the context of Bio OntoCAPE development. The notation used in this section for describing ontologies is resumed in Fig. 3.

The module chemical_process_systems define a chemical process system as a special technical system. The principal classes of the module are chemical process system and process unit, which are modeled as special types of technical systems. All information about a system is obtained via its aspect systems. The aspect system describes the various phases of a system from its design to its operation. See Fig. 3 for a graphical representation of a chemical process system and a process unit, concerning its function and realization.

Consistent with the principles of decomposition of systems, a chemical process system is composed of a certain number of process units. Taking this decomposition into account, the description of a chemical process system can be guessed from the individual description of each of its process units. According to OntoCAPE, a process unit is described by its process steps and its plant items (see Fig. 3). A process step represents a transformation function and a plant item describes where the transformation is physically executed. So, from a functional point of view, a plant item performs a certain process step.

For describing the human endocrine system, we created an individual called endocrineSystem, which represents the endocrine system itself. It is an instance of the biological chemical process system class, which is a subclass of chemical process system, see Fig. 4a. The red rectangles in the figures that we will present all along this section serves to highlight the extensions done to OntoCAPE as well as the instances created in the knowledge base. For this particular example, we have created a subclass chemical process system called biological chemical process system, and then endocrineSystem, as an instance of this last added subclass. 

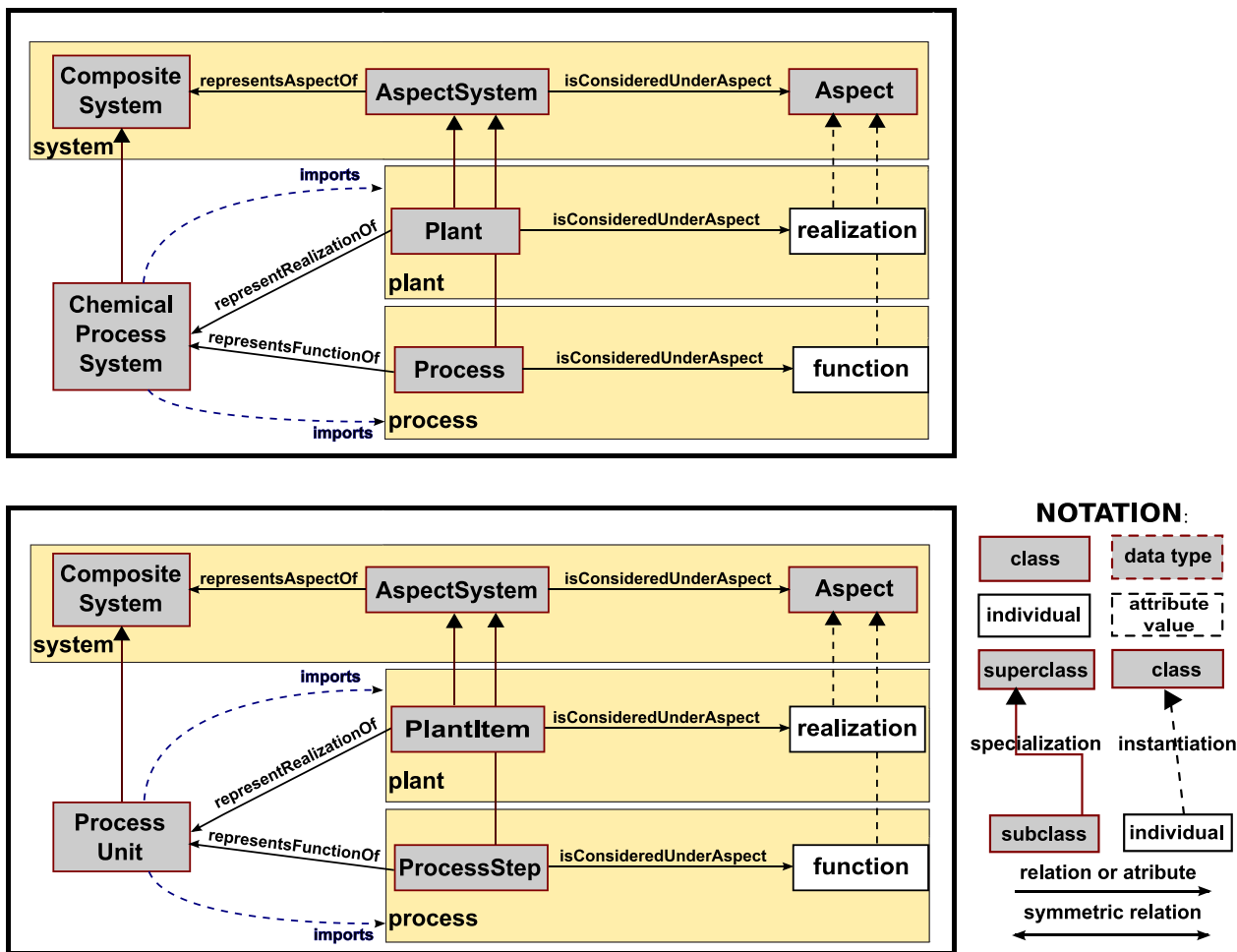

Fig. 3. Presentation of the aspect system considerations for chemical process system and process unit [16, pp. 244, 245].

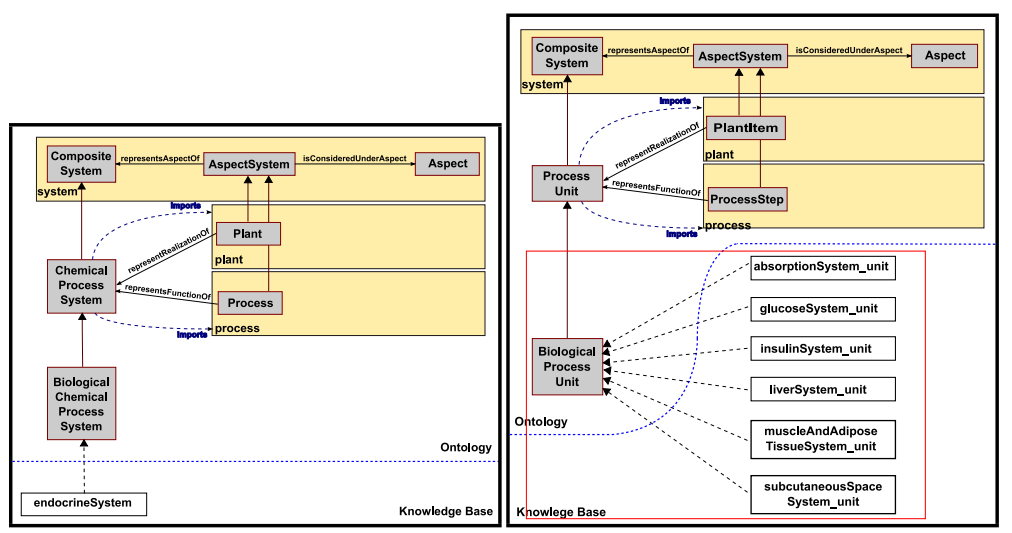

(a) Hierarchy of classes related to (b) Hierarchy of classes related to endocrineSystem the subsystems

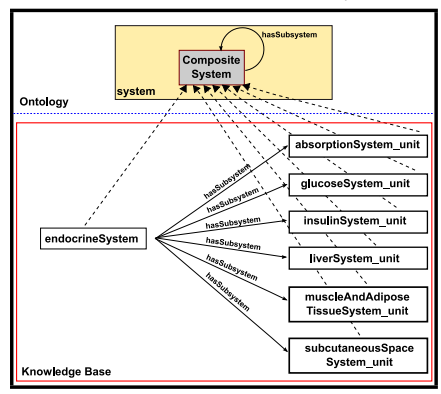

(c) The endocrineSystem related to its subsystems

Fig. 4. Human endrocrine system representation. 


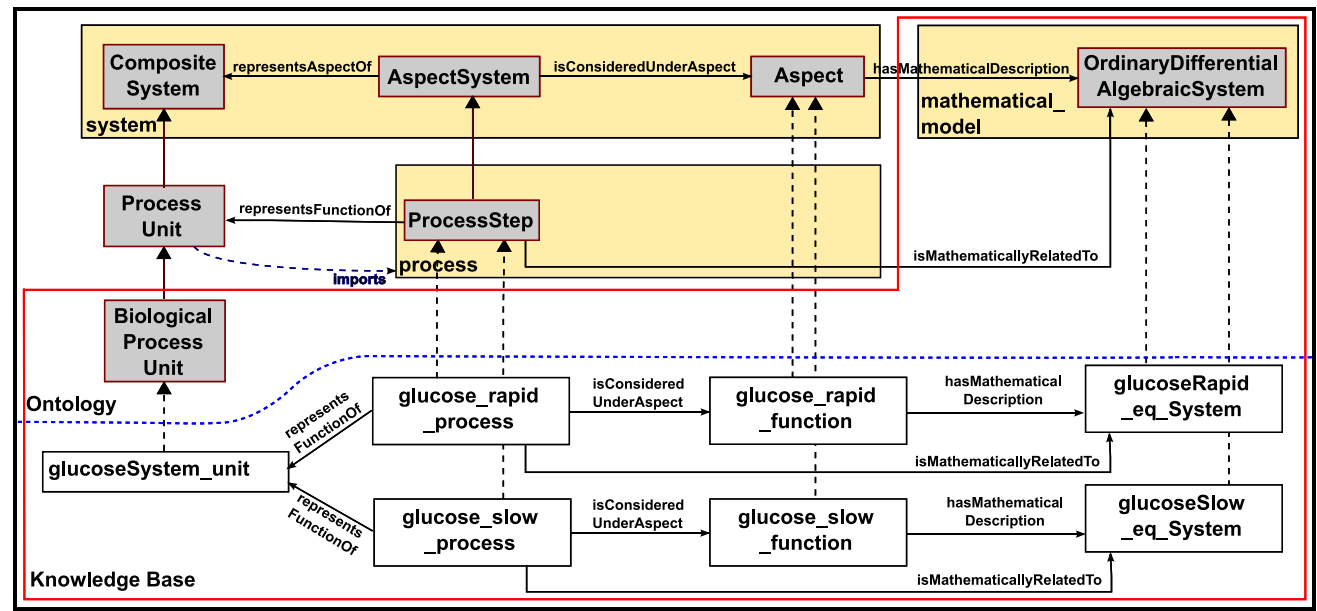

(a) Processes associated to the glucose subsystem function

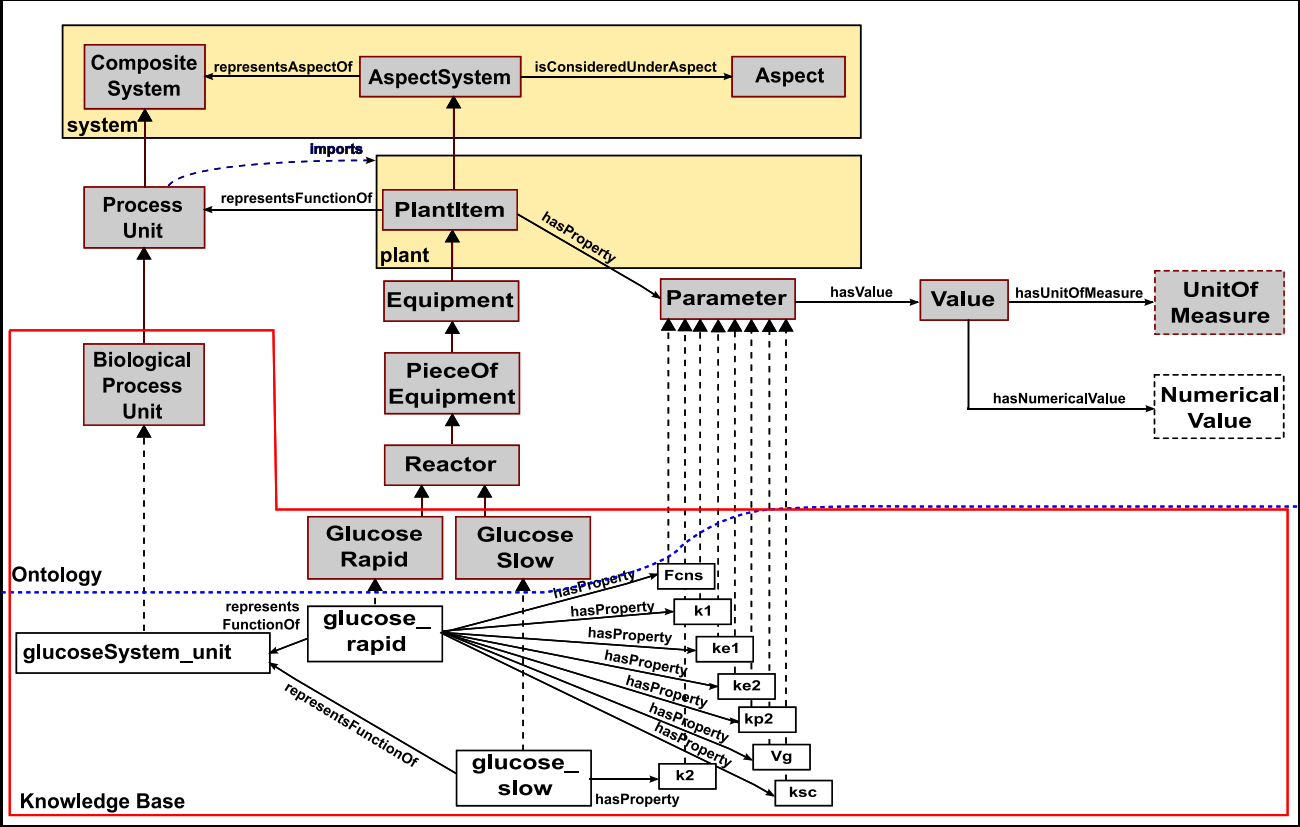

(b) Realization of the glucose subsystem
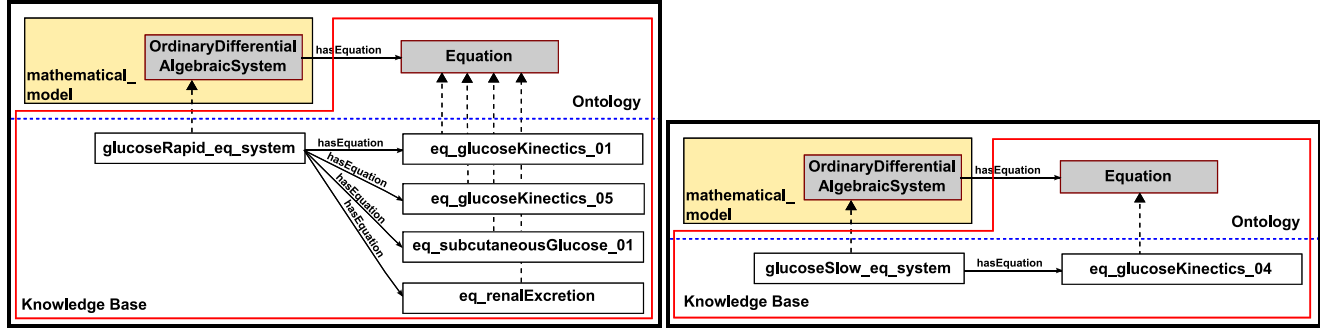

(c) Equations associated to the glucose rapid (d) Equations associated to the glucose slow function function

Fig. 5. Glucose subsystem.

The human endocrine system is composed by six subsystems:

- the glucose,

- the insulin,

- the absorption,

- the liver,

- the muscle and adipose tissue, and

- the subcutaneous space subsystems.

Each subsystem is represented as an instance of the biological process unit class, which is a subclass of process unit.

These instances are named:

- glucoseSystem_unit,

- insulinSystem_unit,

- absorptionSystem_unit, 


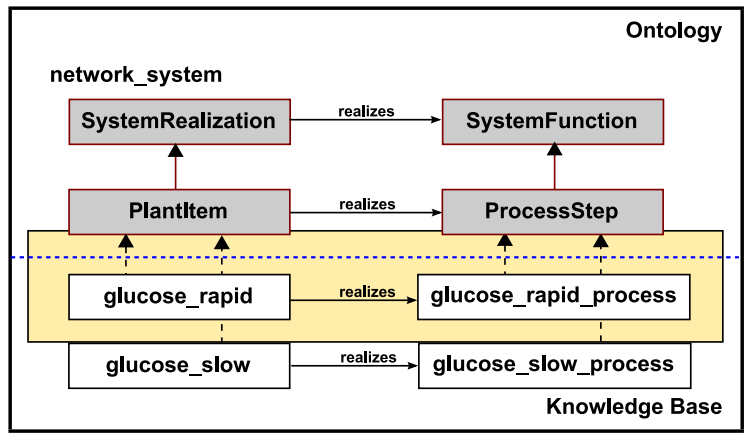

Fig. 6. Glucose subsystem realization connections.

\section{- liverSystem_unit, \\ - muscleAndAdiposeTissueSystem_unit, and \\ - subcutaneousSpaceSystem_unit,}

respectively.

The endocrineSystem relates to all its subsystems through the hasSubsystem Object Property. Figs. 4a, b and c show a graphical representation of the endocrine system and its subsystems.

From now on, we will concentrate and explain in detail the development of the glucose subsystem. The rest of the subsystems were developed analogously and can be consulted in the Appendix B.

Each process unit is composed by process steps. Fig. 5a introduce all process step instances created in the knowledge base that relate to the glucoseSystem_unit. It also show the relationship of each of those process steps to their corresponding biological process unit as well as their function. For example, the glucose_rapid_process is associated to the glucose_rapid_function which can be mathematically described by the ordinary differential algebraic system glucose_rapid_eq_system.

Besides, each process unit is associated to plant items, these plant items represent the equipment where the process step is executed. Fig. 5b introduces all the plant items instances created in the knowledge base that are related to glucoseSystem_unit. These plant items, in fact, are not equipment of some plant but represent some parts of the human body. Some of them can be represented as reactors, other as tanks, depending on their specific function. For example, the glucose_rapid is an instance of the glucose_rapid class, which is a subclass of reactor (we have added this last subclass). This decision has been taken because the glucose_rapid_function is a reaction.

Each plant item has associated some parameters. In the case of the glucose_rapid, it has seven associated parameters: $F_{c n s}, k_{1}, k_{e 1}$, $k_{e 2}, k_{p 2}, V_{g}$ and $k s c$. They must be defined because they are needed by the equations of the model.

Fig. 5c shows that the ordinary differential algebraic system glucose_rapid_eq_system is composed of four equations named eq_glucoseKinectics_01, eq_glucoseKinectics_05, eq_subcutaneousGlucose_01, eq_renalExcretion. These last instances are ontological representations of the UVA/Padova equations (details in Appendix A). Fig. 5d relates the ordinary differential algebraic system glucose_slow_eq_system with its equations.

From the functional point of view, we must indicate which plant item performs which process step. This relationship is explicitly shown for the glucose subsystem in Fig. 6 .

OntoCAPE also provides the necessary concepts for a process description with enough level of detail which is equivalent to that of a process flow diagram. The individual process steps can be con-

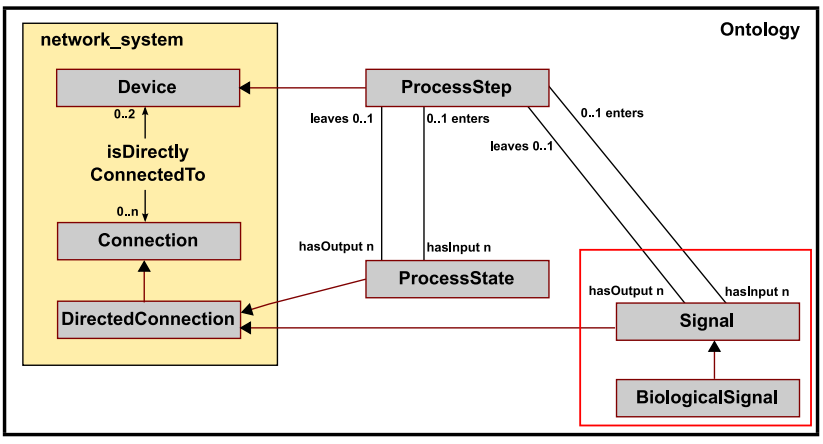

Fig. 7. Ontological representation of process flows. nected to each other via process states. A process state represents the material that enters or leaves a process step. This includes the interchange between two process steps as well as the feeding and removing of material. A process step may have various hasInput and hasOutput relations to a process state (e.g., a reactor that has multiple feeds). A process state, by contrast, must enter and/or leave at most one process step. Even though OntoCAPE had some control schemes, they were not enough to efficiently represent signal flows to complete the closed loop effects. Hence, we have introduced another contribution extending OntoCAPE as shown in Fig. 7.

It is time to introduce the whole mereotopological conception of the human endocrine system. This conception is divided into three diagrams. Two of these diagrams present mass flows of the endocrine system while the third one introduces signal flows. The mass flows correspond to the glucose and insulin flows. The classes corresponding to the individuals are given in brackets. Fig. 8 presents the glucose flows diagram, Fig. 9 describes the insulin flows diagram, whereas Fig. 10 introduces the signal flows diagram, corresponding to the green, blue and red arrows of Fig. 1, respectively.

\section{Interaction between bio ontocape and MATLAB for dynamic simulation and validation}

The creation of the extended knowledge base has been performed using Protégé 4.3. Once OntoCAPE has been extended and the knowledge base representing the human endocrine system has been completely filled in, it was necessary to validate our model.

We developed a java application able to read the ontology and to write and execute a MatLab model. The application developed uses the Jena Ontology API to have access to the ontology and to extract information. Then, it is capable of writing MatLab applications and to run them using the MatLab Control library.

As we already have a computer simulation environment implemented in Simulink ${ }^{\circledR}[12]$ (accepted by the FDA) we decided to use this environment in the validation process. The validation consists on comparing the simulations obtained by the original environment with the simulations obtained using our java application.

Fig. 11 represents graphically the methodology used for validation process. In the upper part of the figure, we represent the simulation process using the UVA/Padova original implementation. In the bottom part of this same figure, we show our java application that extracts information about the human endocrine system from the ontology, then it replaces in the UVA/Padova implementation the module that represents the human endocrine system considering the information obtained from the ontology. Afterwards, the java application runs the simulations.

For doing the validation tests we choose the parameters of 3 adults, one healthy person, one with DMTI and the third with 


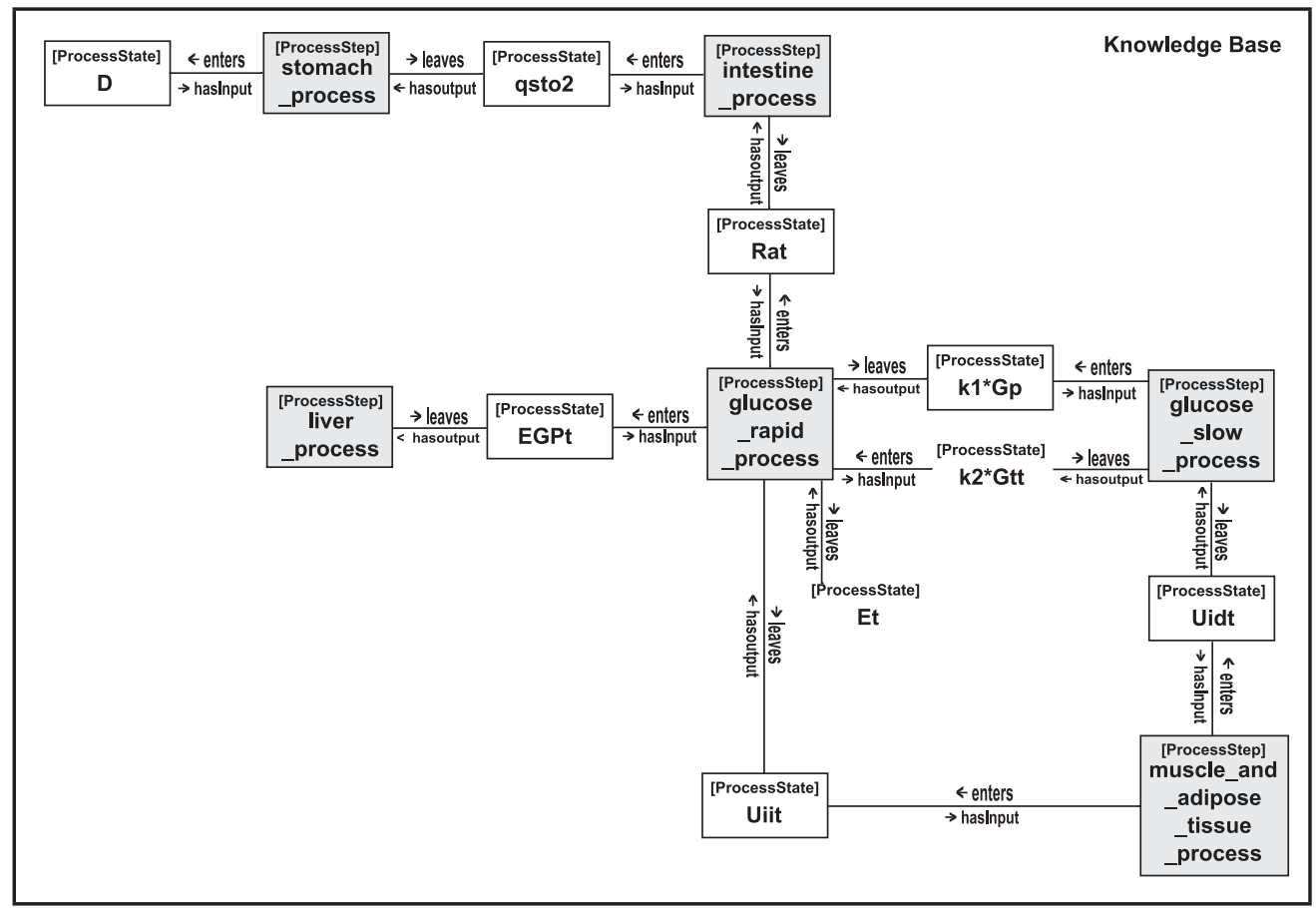

Fig. 8. Glucose flows.

DMTII. We consider that this example represents a good alternative to show that the selected conceptual model for endocrine system is generic enough to be able to analyze different kind of deceases related to regulation problems on blood glucose (glycemia). Since glycemia varies because of different meals intake our experiment consists on giving the same meal to the three subjects at the same time. Since the most important information is related to the quantity of carbohydrates intake with specific meal we detail only the grams of carbohydrates consumed at different hours of the day. The food intake implies the endocrine system will produce the metabolism procedure which will impact on the blood glucose variability. Obviously, the meal intake is taken during certain

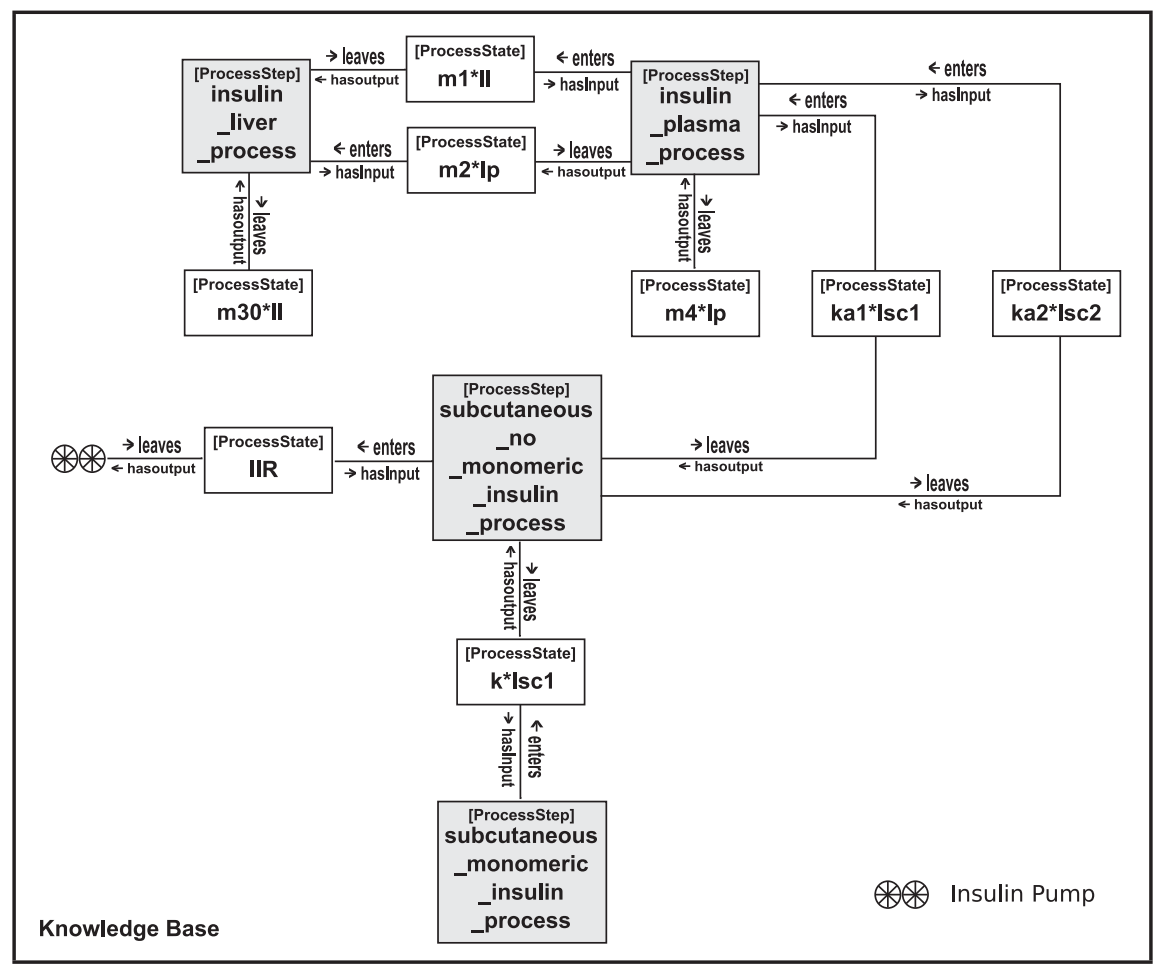

Fig. 9. Insulin flows. 


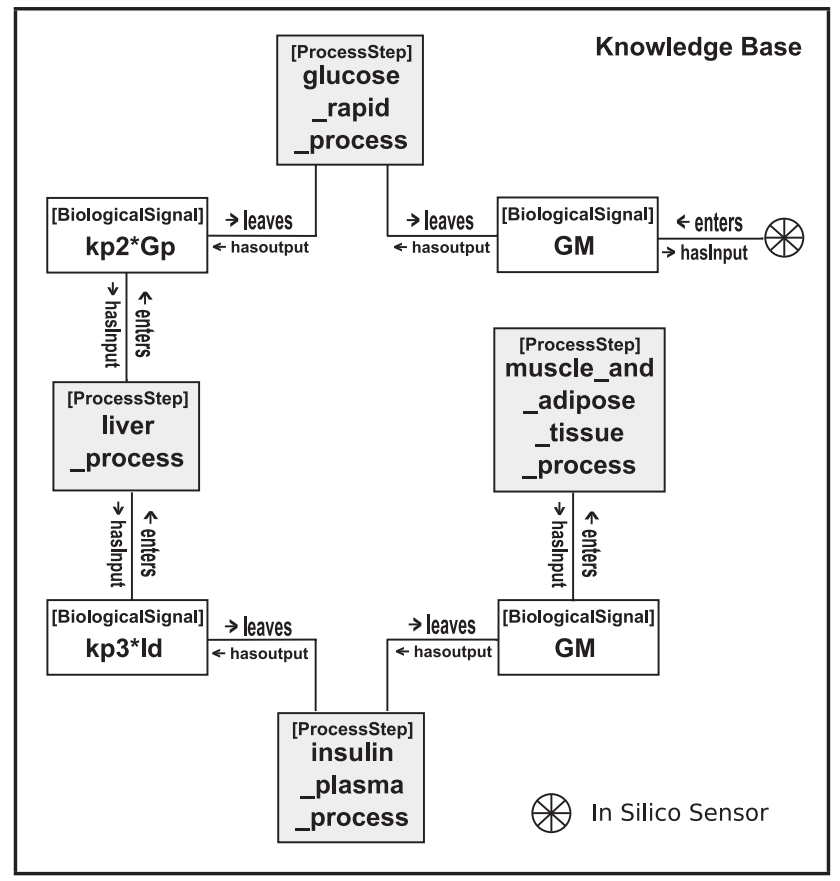

Fig. 10. Signal flows.

period, hence it is represented in a graph where appears the grams of carbohydrates and time required for consumption as a square pulse where the base is the temporal duration and the height is the grams of carbohydrates. A healthy person produces his own insulin to do the metabolism properly, a person with DMTI need to calculate the insulin dosage based on the meal intake and inject it externally because he is practically unable to produce it. A person with DMTII does not have an acceptable response to his own insulin so he must be careful with the diet and physical exercise.

Then, for the three adult subjects we programmed the specific food intake shown in Table 1. At minute 690, the subjects start
Table 1

Food intake in grams of carbohydrates.

\begin{tabular}{lll}
\hline Breakfast & Lunch & Dinner \\
\hline $50 \mathrm{~g}$ & $65 \mathrm{~g}$ & $80 \mathrm{~g}$ \\
\hline
\end{tabular}

having breakfast lasting about 2 min with a carbohydrate content of $50 \mathrm{~g}$. At approximately minute 960, the subjects take a lunch meal containing $65 \mathrm{~g}$ of carbohydrates. The meal duration is about 15 min. At minute 1320, the subjects start taking a dinner meal containing $80 \mathrm{~g}$ of carbohydrates being the meal duration $15 \mathrm{~min}$. The parameters for DMTI correspond to patient 21 [11], who has a body weight of $76.37 \mathrm{~kg}$.

Fig. 12 a shows the blood glucose evolution for the three patients considering the food intake given in Table 1. Besides, Fig. 12b shows the insulin pump action simulated using an artificial pancreas with a predictive functional control (PFC) algorithm to define the insulin dosage for the DMTI patient. For the healthy and DMTII subjects represent the insulin generated by their own body.

Therefore, we demonstrated that, with the same entries for the three subjects we have observed good agreement between both simulations, the original and those obtained by using our java application (which reads our proposed Bio OntoCape). These preliminary results would indicate that we are in the right way with our assumptions about the extension of OntoCape to bioprocess together with its integration with MatLab for temporal evaluations of certain critical variables. The validated results indicate that the ontological representation for the endocrine system is well done and achieve our first objectives of the bigger project for constructing an efficient e-Health system for blood glucose regulation. Concerning clinical implications, it can be seen from the simulations that having good models for each patient is possible to predict wether the insulin dosage is correct or not according to the impact of different meals intake. Hence, the therapists could have an efficient tool for tacking right decisions and make proper recommendations about changes for diet or insulin dosages. Another inherent benefit is that both, patients and therapists, learn more about each diabetic person endocrine system behavior and it drives to better treatments for handling well this disease.

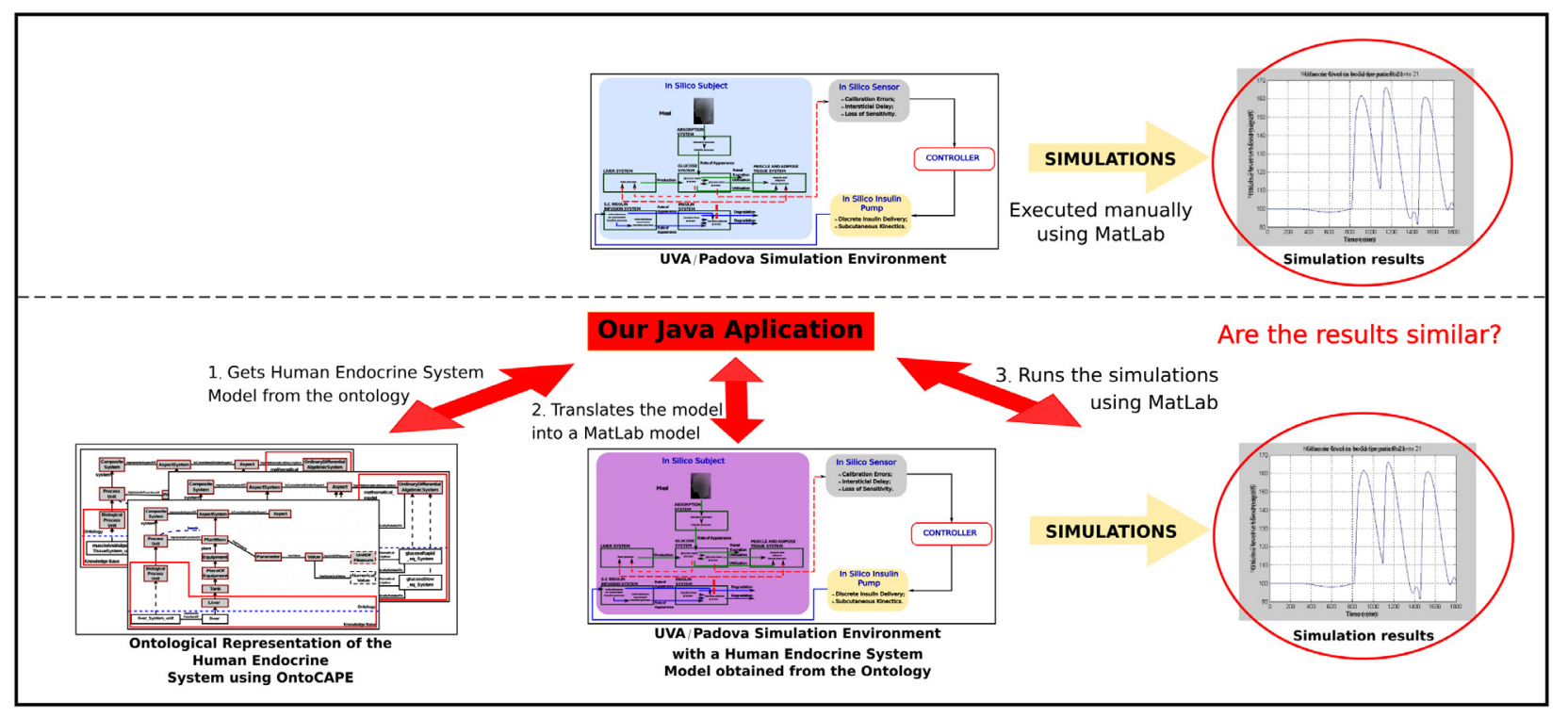

Fig. 11. Validation process. 


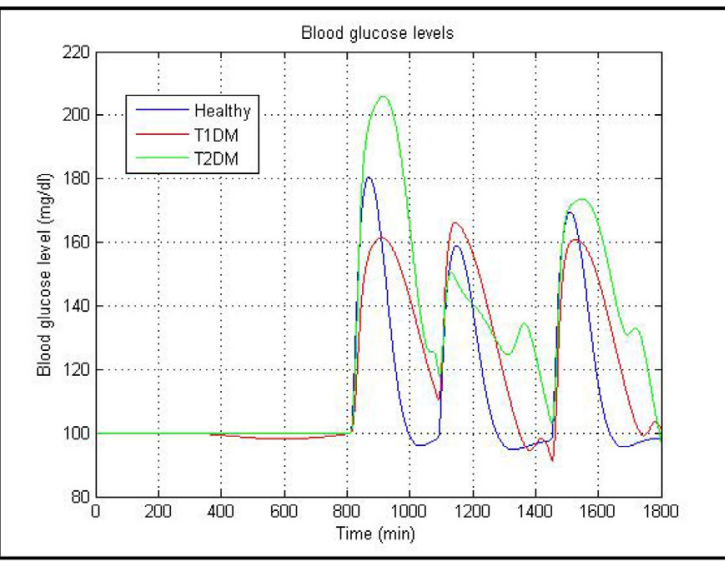

(a) Simulation of glucose level evolution in blood

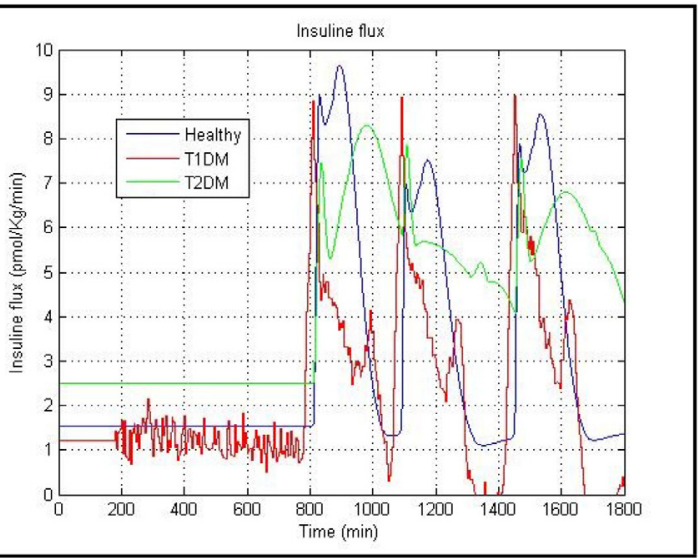

(b) Simulation of insulin action

Fig. 12. Simulation of patients.

\section{Conclusions and future work}

In this work we presented an efficient way for using and extending an existing ontology for developing our Bio OntoCAPE and achieving one of the main objective of our project. We detailed how to incorporate expert knowledge of a domain in a way that computers can 'understand' and 'reason about this'. To propose an ontological representation of the human endocrine system, we have deeply studied the OntoCAPE ontology and the methodology for obtaining mathematical models of biological systems. This ontology is a general-purpose one for applications in the domain of computer-aided bio process engineering, which focuses on complex bio chemical process systems. In addition it considers the use of devices such as sensors, valves, pumps and allows taking into account control algorithms which are helpful for several deceases. This is an important issue since pathological problems associated with deceases like DMTI needs some or all of those elements to be properly treated. Our first fundamental assumption is that the human endocrine system is a chemical process system too, that was the reason of why we considered OntoCAPE as a good starting point. Therefor, we are confident to have demonstrated through the three cases presented here that this is a valid argument. In this work, we described the way in which we captured the expert knowledge for constructing mathematical models of the human endocrine system healthy and with some pathologies and the used tools for transforming that knowledge in an ontological representation. By applying this methodology we believe that we have generated the first version of an ontology dedicated to model bio process using rigorous engineering concepts. The development of this first part of Bio OntoCAPE consisting of the ontological representation of the human endocrine system was performed having into account the future integration with other ontologies to assist a multidisciplinary staff with different levels of expertise. This procedure was followed having in mind the main objective of conforming an e-Health platform for diabetes care.

The main disadvantage of using OntoCAPE was its size (composed of 62 files). It was very difficult to use the reasoners because it took an average of three hours to check consistency. We are planning to recreate the work done using OntoCAPE to develop our own ontology. This would enable us to use reasoners to infer logical consequences from a set of asserted facts and axioms, in an easier way. Another disadvantage could be considered the inherent difficulties of representing the human body behavior through the use of phenomenological models. In this context, we found in the literature some interesting works where the predictive models are obtained through time series decomposition, see for example [22]. Hence, in future we plan the use of data from diabetic patients to construct efficient models for predicting the responses when specific meals intake and insulin dosage are given.

One advantage of the work done is that we were able to cope with time by connecting our Bio OntoCAPE with MatLab platform using the java application developed, we were able to simulate patients evolution over time. This is an important issue since we need to know how to construct this kind of biological models but through their computer implementations we are able to predict in certain time horizon the dynamic behavior of the most critical variables. In this context, we have in mind as future work to analyze if it could be necessary to develop a new ontology based on the ideas of [21] who presented an ontology-based framework to support intelligent data analysis of recorded temporal data. They have shown how a process condition can be inferred when qualitative temporal patterns are available. These skills are very useful for doing recommendations each time the patient is out of the healthy range of glycemia. Another possibility is to take into account the work of [17] who proposed a fuzzy framework for encoding uncertainty in clinical decision-making to analyze insulin dosage and meals and recommend corrections to both.

Other planned topic to do in the next future involves the development of the ontological representation of control system, towards the implementation of the artificial pancreas for patients with DMTI. Hence, the insulin dosage can be suggested by running a control algorithm, specially designed for each patient accounting specific external stimuli such as meals intake and insulin. Besides, we are working on a nutritional ontology, thought to be integrated with our Bio OntoCape which will contain information about food as well as useful rules for recommending to diabetic patients to have a proper daily diet.

\section{Appendix A. Mathematical representation of the human endocrine system}

In this section, we present a detailed explanation of the UVA/Padova model equations.

The behavior of the subsystems considered in Fig. 1 are described by the following Eq. [5]:

$$
\begin{aligned}
& \dot{G}_{p}(t)=E G P(t)+R a(t)-U_{i i}(t)-E(t)-k_{1} G_{p}(t)+k_{2} G_{t}(t) \\
& \dot{G}_{t}(t)=-U_{i d}(t)+k_{1} G_{p}(t)-k_{2} G_{t}(t)
\end{aligned}
$$


Table A.2

Variables and parameters used in the UVA/Padova glucose-insulin system [5, p. 29].

\begin{tabular}{|c|c|}
\hline Variable/parameter & Unit \\
\hline$G_{p}$ : Glucose mass in plasma and rapidly equilibrating tissues. & {$[\mathrm{mg} / \mathrm{kg}]$} \\
\hline$G_{t}:$ Glucose mass in slowly equilibrating tissues. & {$[\mathrm{mg} / \mathrm{kg}]$} \\
\hline G: Plasma glucose concentration. & {$[\mathrm{mg} / \mathrm{dl}]$} \\
\hline$G_{M}$ : Plasma glucose concentration measured subcutaneously. & {$[\mathrm{mg} / \mathrm{kg}]$} \\
\hline EGP: Endogenous glucose production. & {$[\mathrm{mg} / \mathrm{kg} / \mathrm{min}]$} \\
\hline$k_{p 1}$ : Extrapolated EGP at zero glucose and insulin. & {$[\mathrm{mg} / \mathrm{kg} / \mathrm{min}]$} \\
\hline$k_{p 3}$ : Parameter governing amplitude of insulin action on the liver. & {$[\mathrm{mg} / \mathrm{kg} / \mathrm{min}]$ per $[\mathrm{pmol} / \mathrm{l}]$} \\
\hline$R_{a}:$ Glucose rate of appearance in plasma. & {$[\mathrm{mg} / \mathrm{kg} / \mathrm{min}]$} \\
\hline E: Renal excretion. & {$[\mathrm{mg} / \mathrm{kg} / \mathrm{min}]$} \\
\hline$k_{e 1}:$ Glomerular filtration rate. & {$\left[\mathrm{min}^{-1}\right]$} \\
\hline$k_{e 2}$ : Renal threshold of glucose. & {$[\mathrm{mg} / \mathrm{kg}]$} \\
\hline$U_{i i}$ and $U_{i d}$ : Insulin independent and dependent glucose utilisations. & {$[\mathrm{mg} / \mathrm{kg} / \mathrm{min}]$} \\
\hline$V_{m}(X)$ : Parameter from the Michaelis Menten equation. & {$[\mathrm{mg} / \mathrm{kg} / \mathrm{min}]$} \\
\hline$K_{m 0}$ : Parameter from the Michaelis Menten equation. & {$[\mathrm{mg} / \mathrm{kg}]$} \\
\hline$p_{2 U}$ : Rate constant of insulin action on the peripheral glucose utilisation. & {$\left[\mathrm{min}^{-1}\right]$} \\
\hline$F_{\text {cns }}$ : Glucose uptake by the brain and erythrocytes. & {$[\mathrm{mg} / \mathrm{kg} / \mathrm{min}]$} \\
\hline$k_{1}$ and $k_{2}:$ Rate parameters. & {$\left[\mathrm{min}^{-1}\right]$} \\
\hline$V_{G}$ : Distribution volume of glucose. & {$[\mathrm{dl} / \mathrm{kg}]$} \\
\hline$I_{p}:$ Insulin mass in plasma. & {$[\mathrm{pmol} / \mathrm{kg}]$} \\
\hline I: Insulin mass in liver. & {$[\mathrm{pmol} / \mathrm{kg}]$} \\
\hline I: Plasma insulin concentration. & {$[\mathrm{pmol} / \mathrm{l}]$} \\
\hline$V_{I}$ : Distribution volume of insulin. & {$[\mathrm{l} / \mathrm{kg}]$} \\
\hline HE: Hepatic extraction of insulin. & Dimensionless \\
\hline$m_{1}, m_{2}, m_{3}$ and $m_{4}:$ Rate parameters. & {$\left[\min ^{-1}\right]$} \\
\hline$I_{d}:$ Delayed insulin signal. & {$[\mathrm{pmol} / \mathrm{l}]$} \\
\hline$I_{1}$ : Insulin signal associated with $I_{d}$. & [pmol/l] \\
\hline$k_{i}$ : Rate parameter accounting for delay between insulin signal and insulin action. & {$\left[\mathrm{min}^{-1}\right]$} \\
\hline$R_{i}$ : Rate of appearance of insulin in plasma. & {$[\mathrm{pmol} / \mathrm{kg} / \mathrm{min}]$} \\
\hline$k_{d}, k_{a 1}$ and $k_{a 2}$ : Rate parameters accounting for subcutaneous insulin kinetics. & {$\left[\min ^{-1}\right]$} \\
\hline$X:$ Insulin in the interstitial fluid. & {$[\mathrm{pmol} / \mathrm{l}]$} \\
\hline
\end{tabular}

Table A.3

Variables and parameters used in the UVA/Padova glucose absorption model [5, p. 30].

\begin{tabular}{ll}
\hline Variable/parameter & Unit \\
\hline$Q_{s t o}:$ Total amount of glucose in the stomach & {$[\mathrm{mg}]$} \\
$Q_{s t 01}:$ Amount of glucose in the stomach (solid phase) & {$[\mathrm{mg}]$} \\
$Q_{s t o 2}:$ Amount of glucose in the stomach (triturated phase) & {$[\mathrm{mg}]$} \\
$Q_{\text {gut }}:$ Amount of glucose in the intestine & {$[\mathrm{mg}]$} \\
$D:$ Ingested glucose dose & {$[\mathrm{mg}]$} \\
$k_{\text {gri }}:$ Rate of grinding. & {$\left[\mathrm{min}^{-1}\right]$} \\
$k_{\text {abs }}:$ Rate of intestinal absorption. & {$\left[\mathrm{min}^{-1}\right]$} \\
$k_{\text {empt }}:$ Rate of gastric emptying. & {$\left[\mathrm{min}^{-1}\right]$} \\
$k_{\text {max }}:$ Maximum rate of gastric emptying. & {$\left[\mathrm{min}^{-1}\right]$} \\
$k_{\min }:$ Minimum rate of gastric emptying. & {$\left[\mathrm{min}^{-1}\right]$} \\
$b:$ Percentage of the dose for which $k_{\text {empt }}$ decreases $t o\left(k_{\max }-k_{\min }\right) / 2$. & Dimensionless \\
$c:$ Percentage of the dose for which $k_{\text {empt }}$ is back to $t o\left(k_{\max }-k_{\min }\right) / 2$. & Dimensionless \\
$f:$ Fraction of the intestinal absorption which appears in plasma. & Dimensionless \\
$B W:$ Body weight. & {$\left[\mathrm{kg}^{2}\right.$} \\
\hline
\end{tabular}

$G(t)=\frac{G_{p}(t)}{V_{G}}$

$\dot{G_{M}}(t)=k_{s c} G_{p}(t)-k_{s c} G_{M}(t)$

$E G P(t)=-k_{p 1}-k_{p 2} G_{p}(t)-k_{p 3} I_{d}(t)$

$U_{i i}(t)=F_{c n s}$

$U_{i d}(t)=\frac{V_{m}(X) G_{t}(t)}{K_{m 0}+G_{t}(t)}$

$E(t)=\left\{\begin{aligned} k_{e 1}\left[G_{p}(t)-k_{e 2}\right], & G_{p}(t)>k_{e 2} \\ 0, & G_{p}(t) \leq k_{e 2}\end{aligned}\right.$

For the insulin and the subcutaneous insulin infusion subsystems, represented in Fig. 1, the corresponding equations are [5]:

$\dot{I}(t)=-\left(m_{1}+m_{3}\right) I(t)+m_{2} I_{p}(t)$

$$
\begin{aligned}
& \dot{I}_{p}(t)=-\left(m_{2}+m_{4}\right) I_{p}(t)+m_{1} I(t)+R_{i}(t) \\
& I(t)=\frac{I_{p}(t)}{V_{I}} \\
& m_{3}=\frac{H E_{b} m_{1}}{1-H E_{b}} \\
& \dot{I}_{1}(t)=-k_{1}\left(I_{1}-I\right)(t) \\
& \dot{I}_{d}(t)=-k_{i}\left(I_{d}-I_{1}\right)(t) \\
& V_{m 0}=\frac{\left(E G P_{b}-F c n s\right)\left(K_{m 0}+G_{t b}\right)}{G_{t b}} \\
& V_{m}(X)=V_{m 0}+V_{m X} X(t)
\end{aligned}
$$

$$
\dot{X}(t)=-p_{2 U} X(t)+p_{2 U}\left[I(t)-I_{b}\right]
$$

$$
\dot{I}_{s c 1}=-\left(k_{d}+k_{a 1}\right) I_{s c 1}(t)+I I R(t)
$$


$\dot{I}_{s c 2}=k_{d} I_{s c 1}(t)-k_{a 2} I_{s c 2}(t)$

$R_{i}(t)=k_{a 1} I_{s c 1}(t)+k_{a 2} I_{s c 2}(t)$

Note that suffix $b$ denotes basal state. The model notation is reported in Table A.2.

The glucose absorption model is presented in [15] and illustrated in Fig. 1.

The key feature of this model is that the gastric emptying rate $\left(k_{\text {empt }}\right)$ is described realistically, because it depends nonlinearly on the amount of glucose in the stomach $\left(Q_{s t o}\right)$ as shown in the following equations:

$Q_{s t o}(t)=Q_{s t o 1}(t)+Q_{s t o 2}(t)$

$\dot{Q}_{s t o 1}(t)=-k_{\text {gri }} Q_{s t o 1}(t)+D \delta(t)$

$\dot{Q}_{s t o 2}(t)=-k_{\text {empt }}\left(Q_{s t o}\right) Q_{s t o 2}(t)+k_{\text {gri }} Q_{s t o 1}(t)$

$\dot{Q}_{\text {gut }}(t)=-k_{\text {asb }} Q_{\text {gut }}(t)+k_{\text {empt }}\left(Q_{s t o}\right) Q_{s t o 2}(t)$

$k_{\text {empt }}\left(Q_{\text {sto }}\right)=k_{\text {min }}+\frac{k_{\text {max }}-k_{\min }}{2}\left\{\tanh \left[\alpha\left(Q_{\text {sto }}-b D\right)\right]\right.$

$$
\left.-\tanh \left[\beta\left(Q_{s t o-c D}\right)\right]+2\right\}
$$

$\alpha=\frac{5}{2 D(1-b)}, \beta=\frac{5}{2 D c}$

$R a(t)=\frac{f k_{a b s} Q_{\text {gut }}}{B W}$

The model notation is reported in Table A.3.

\section{Appendix B. Ontological model}

This section completes the ontological model of the human endocrine system. Fig. B.13a shows the binary tree structure used for representing equations. Fig. B.13b shows how we represent Eq. (A.21) using this structure.
Table B.4

Relation between equations in the knowledge base and the mathematical model.

\begin{tabular}{ll}
\hline $\begin{array}{l}\text { Ontological representation (equation } \\
\text { instances) }\end{array}$ & $\begin{array}{l}\text { UVA/Padova } \\
\text { equations (Section 3) }\end{array}$ \\
\hline eq_stomach_01 & Eq. (A.21) \\
eq_stomach_02 & Eq. (A.22) \\
eq_stomach_03 & Eq. (A.26) ( $\alpha$ ) \\
eq_stomach_04 & Eq. (A.26) ( $\beta$ ) \\
eq_stomach_05 & Eq. (A.25) \\
eq_stomach_06 & Eq. (A.23) \\
eq_intestine_01 & Eq. (A.24) \\
eq_intestine_02 & Eq. (A.27) \\
eq_glucoseKinetics_01 & Eq. (A.1) \\
eq_glucoseKinetics_05 & Eq. (A.3) \\
eq_subcutaneousGlucose_01 & Eq. (A.4) \\
eq_renalExcretion & Eq. (A.8) \\
eq_glucoseKinetics_04 & Eq. (A.2) \\
eq_insulinInTheLiver_01 & Eq. (A.9) \\
eq_liver_01 & Eq. (A.5) \\
eq_insulinActionOnProduction_01 & Eq. (A.13) \\
eq_insulinActionOnProduction_02 & Eq. (A.14) \\
eq_insulinActionOnGlucoseUtilization_01 & Eq. (A.17) \\
eq_insulinKinetics_01 & Eq. (A.10) \\
eq_insulinKinetics_02 & Eq. (A.11) \\
eq_glucoseUtilization_01 & Eq. (A.6) \\
eq_glucoseKinetics_02 & Eq. (A.16) \\
eq_glucoseKinetics_03 & Eq. (A.7) \\
eq_subcutaneousInsulinKinetics_01 & Eq. (A.18) \\
eq_subcutaneousInsulinKinetics_02 & Eq. (A.19) \\
\hline
\end{tabular}

Table B.4 relates the equation instances in the knowledge base with the equations presented by the mathematical model UVA/Padova.

Figs. B.14-B.18 present the development of the absorption, the insulin, the liver, the muscle and adipose tissue and the subcutaneous space subsystems, respectively.

Finally, Fig. B.19 completes the model from the functional point of view. It indicates which plant item performs which process step.

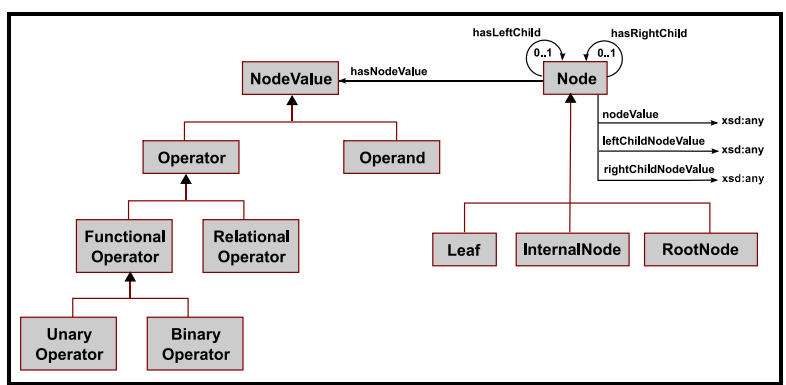

(a) Binary tree structure [16, p. 164]

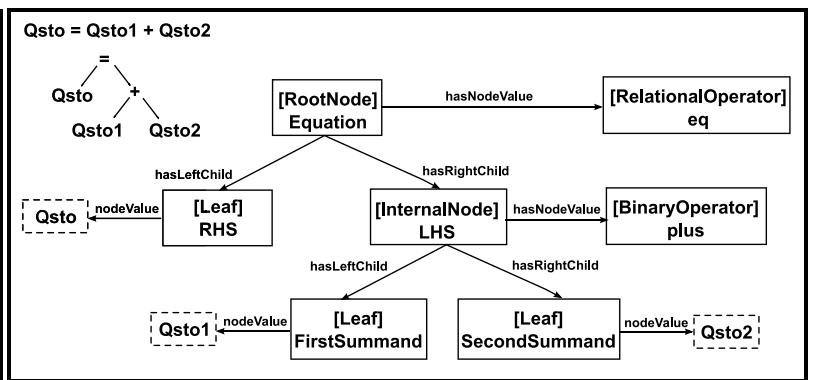

(b) Representing an equation using binary tree structure

Fig. B.13. Ontological representation of equations, 


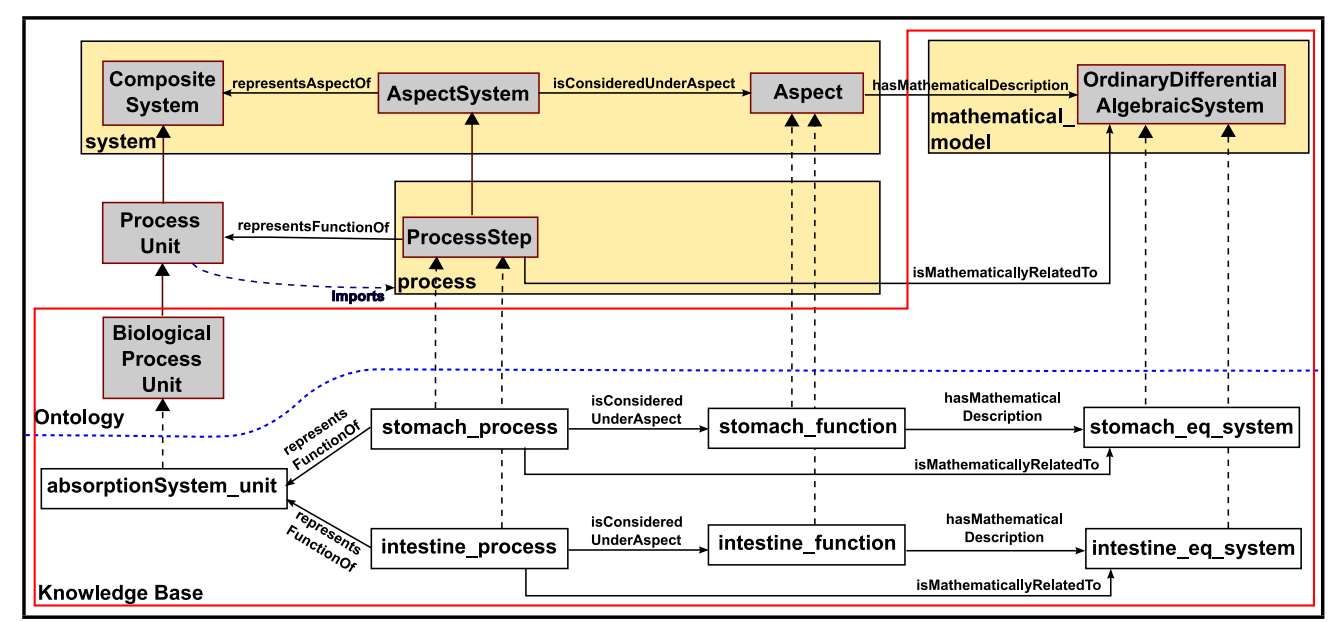

(a) Processes associated to the absorption subsystem function

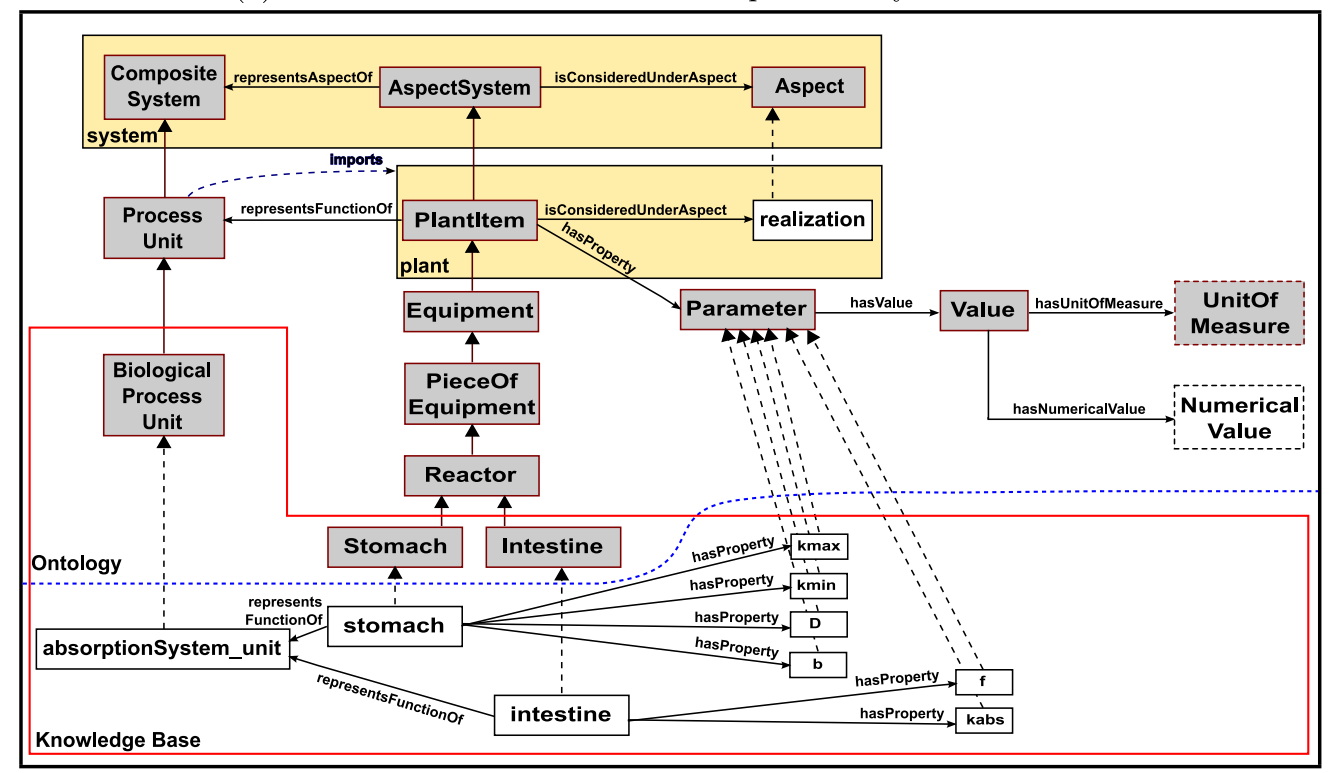

(b) Realization of the absorption subsystem

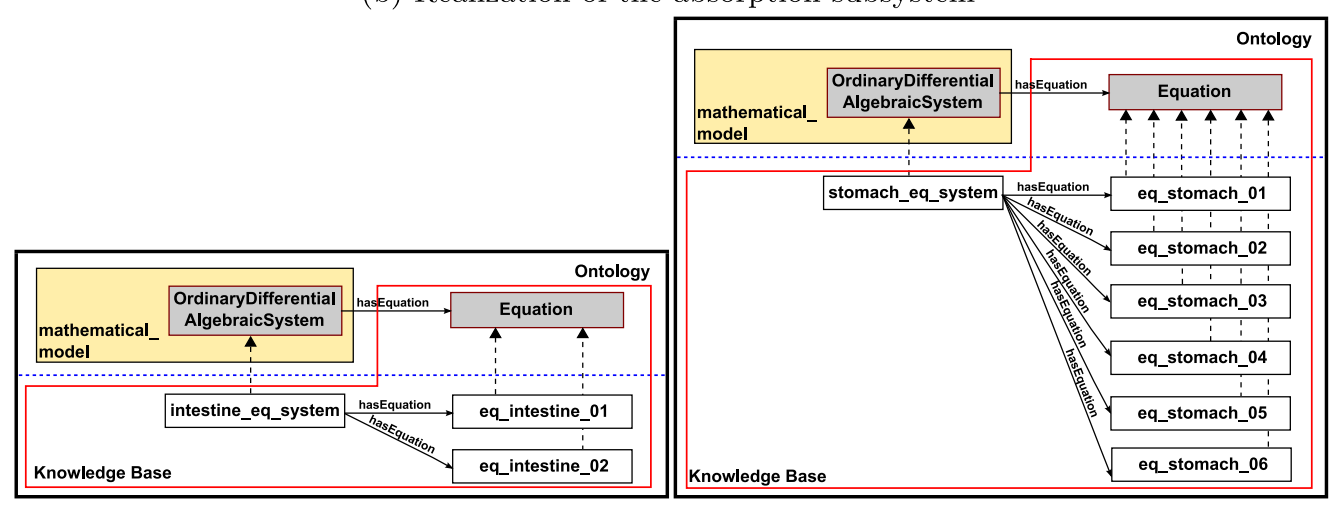

(c) Equations associated to the intestine func- (d) Equations associated to the stomach function tion

Fig. B.14. Absorption subsystem. 


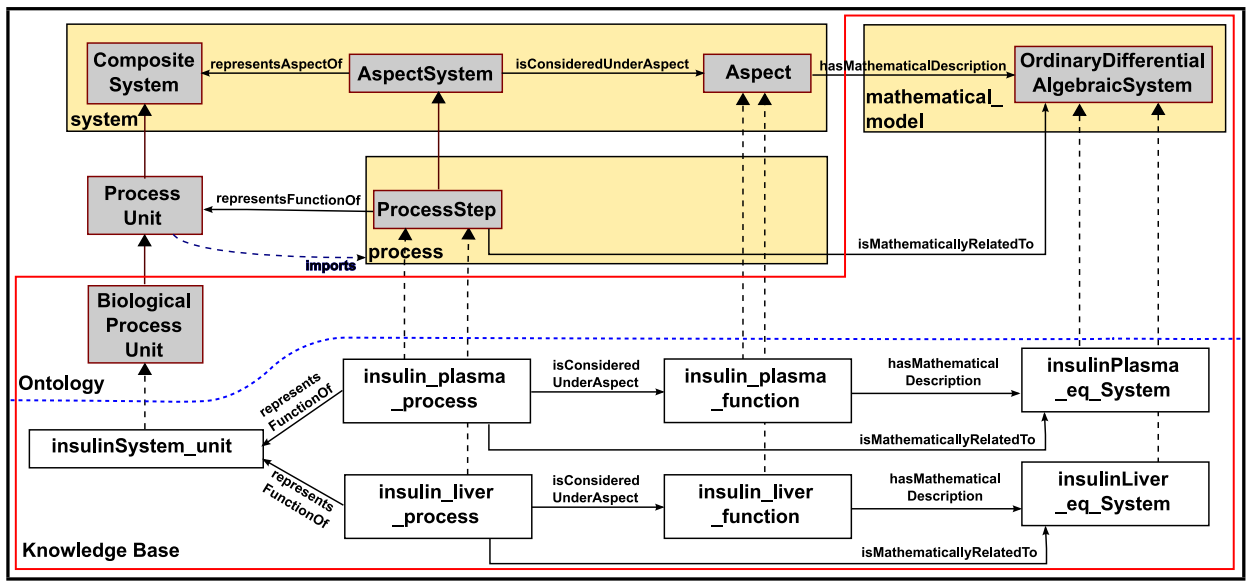

(a) Processes associated to the insulin subsystem function

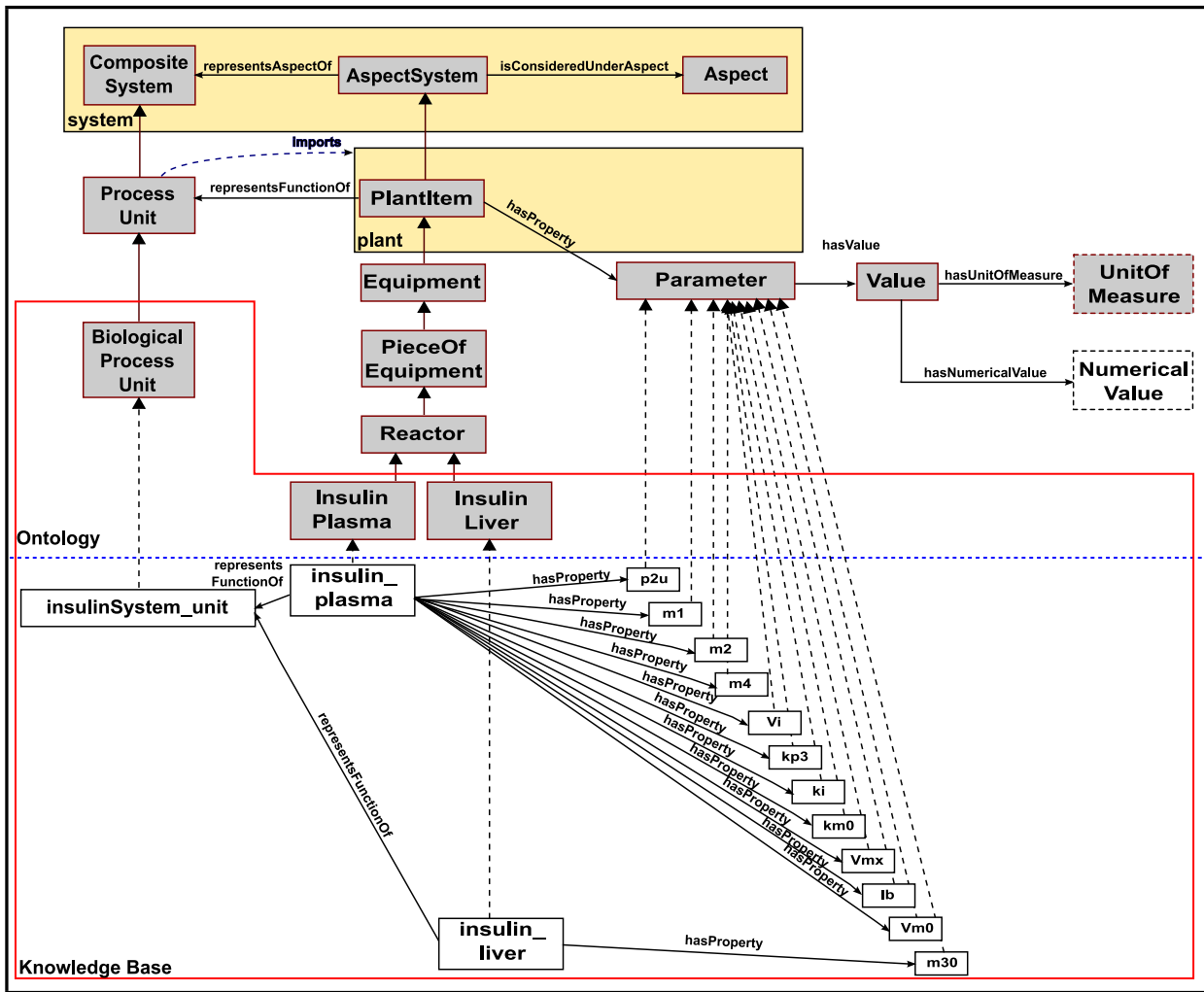

(b) Realization of the insulin subsystem

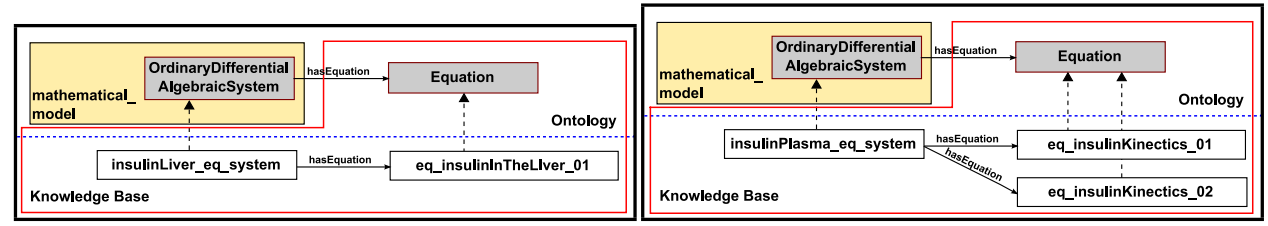

(c) Equations associated to the insulin liver (d) Equations associated to the insulin plasma function function

Fig. B.15. Insulin subsystem. 


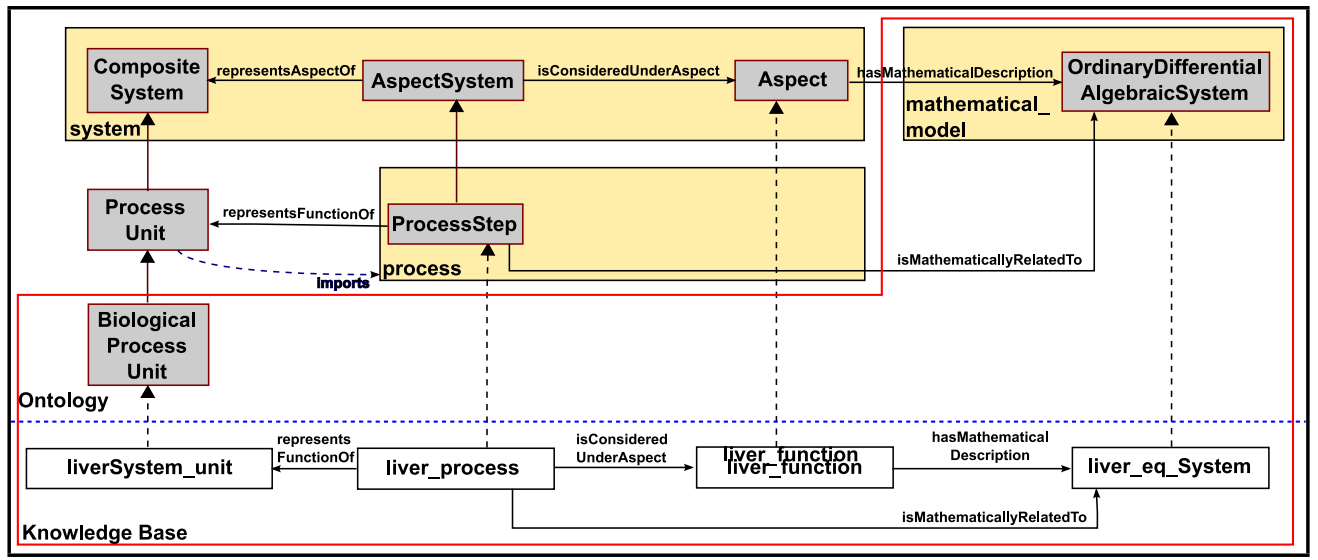

(a) Processes associated to the liver subsystem function

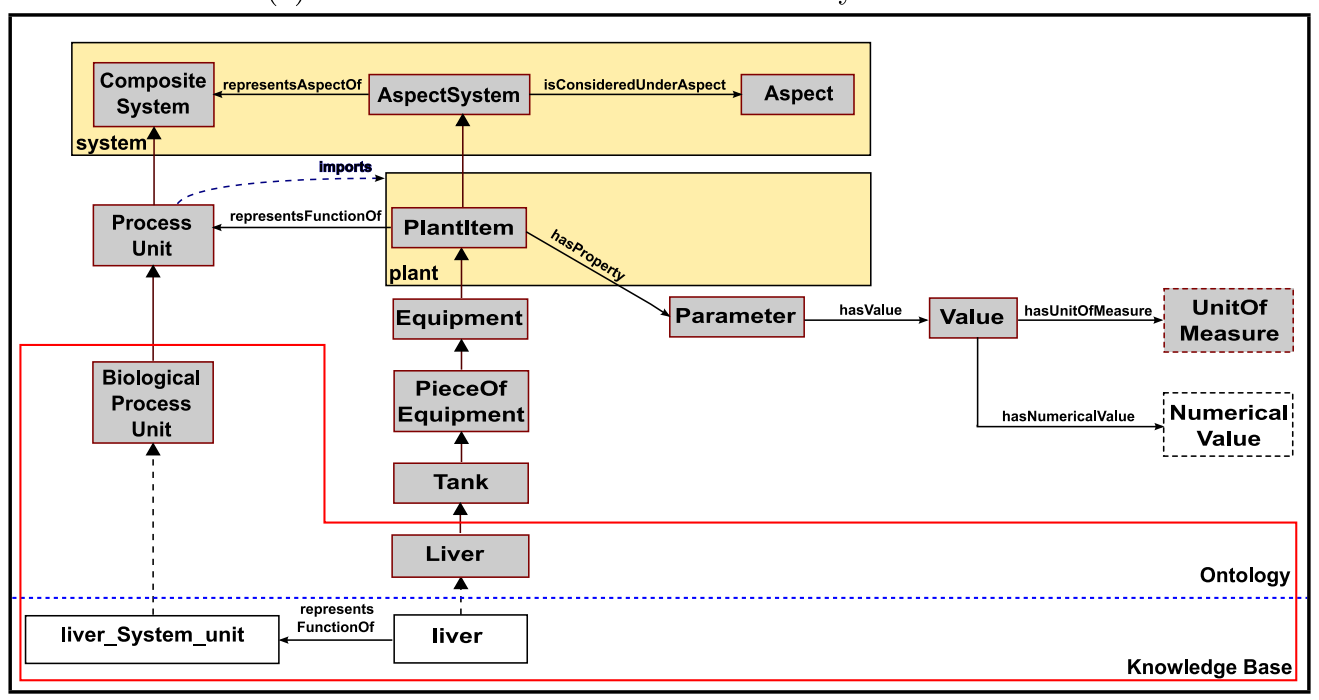

(b) Realization of the liver subsystem

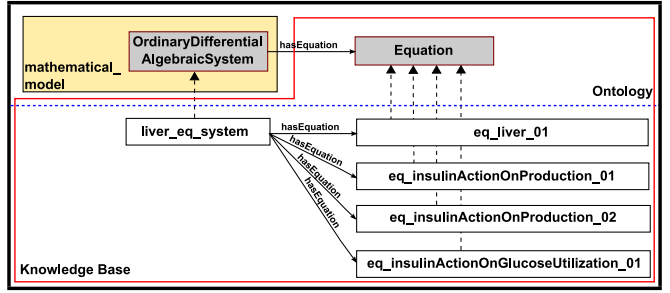

(c) Equations associated to the liver function

Fig. B.16. Liver subsystem. 


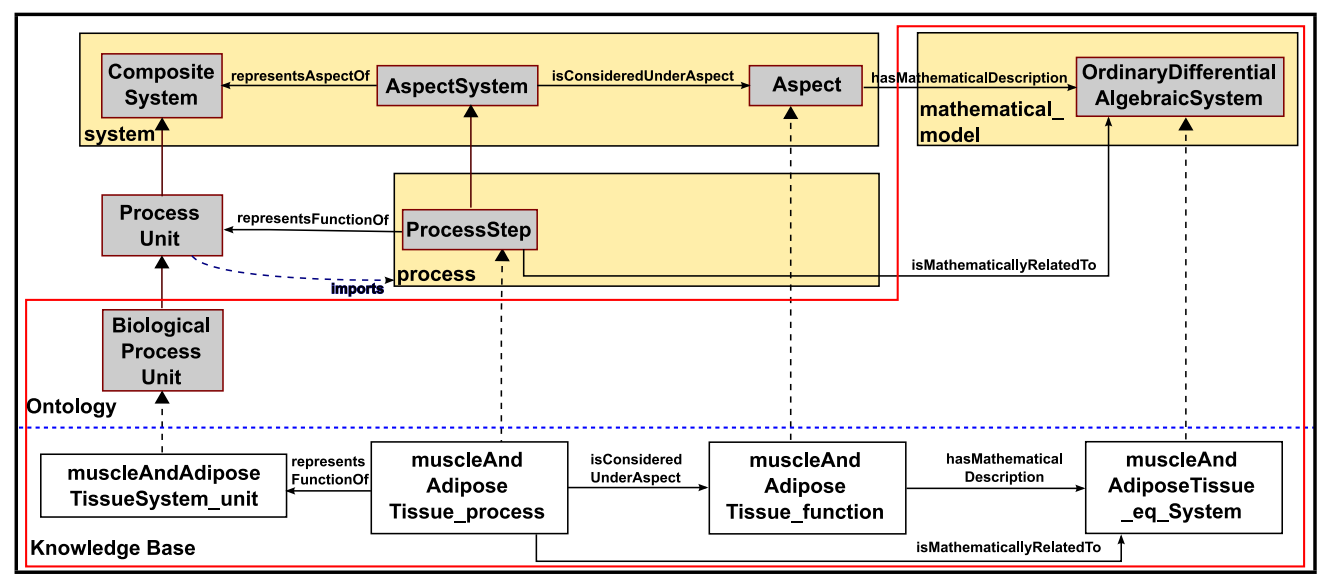

(a) Process associated to the muscle and adipose tissue subsystem function

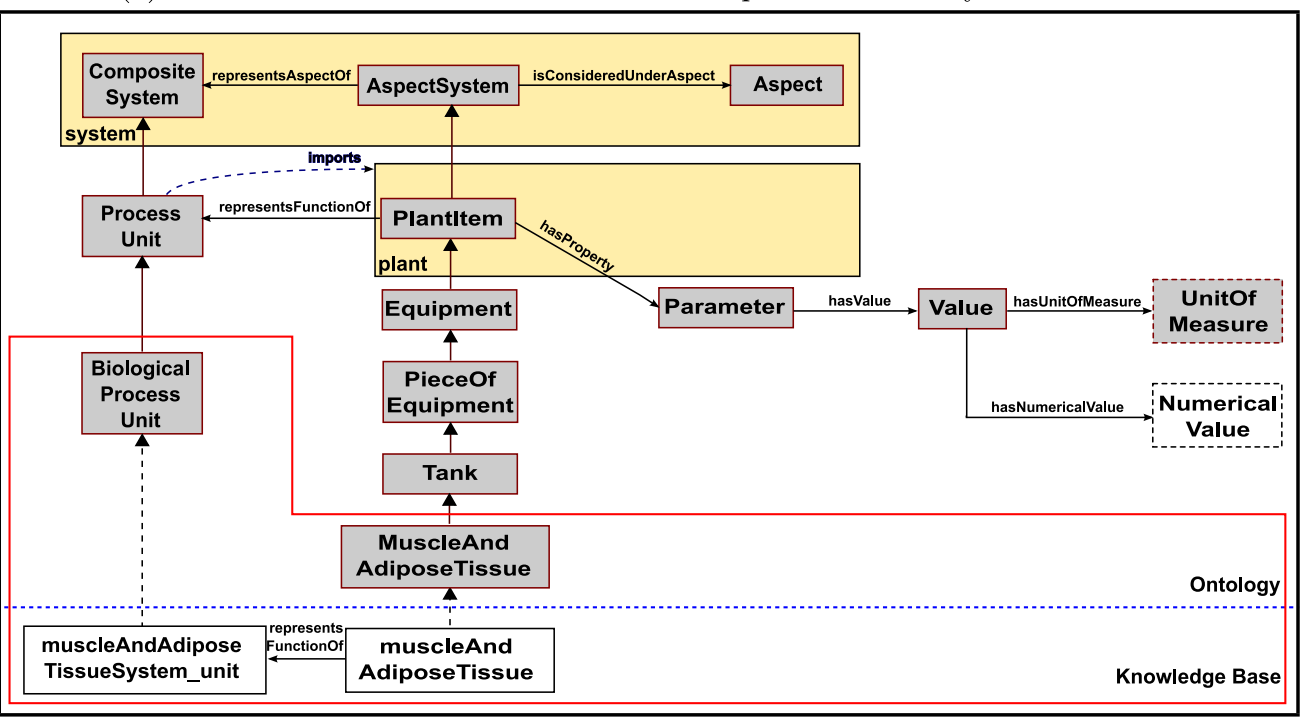

(b) Realization of the muscle and adipose tissue subsystem

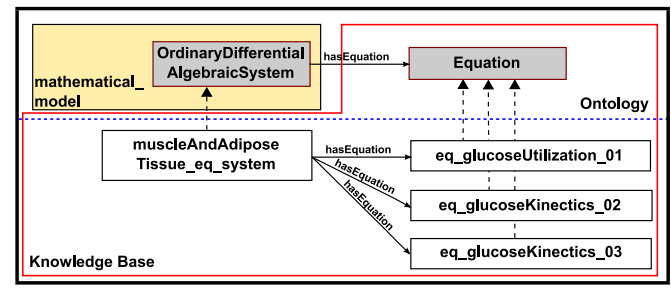

(c) Equations associated to the muscle and adipose tissue subsystem function

Fig. B.17. Muscle and adipose tissue subsystem. 


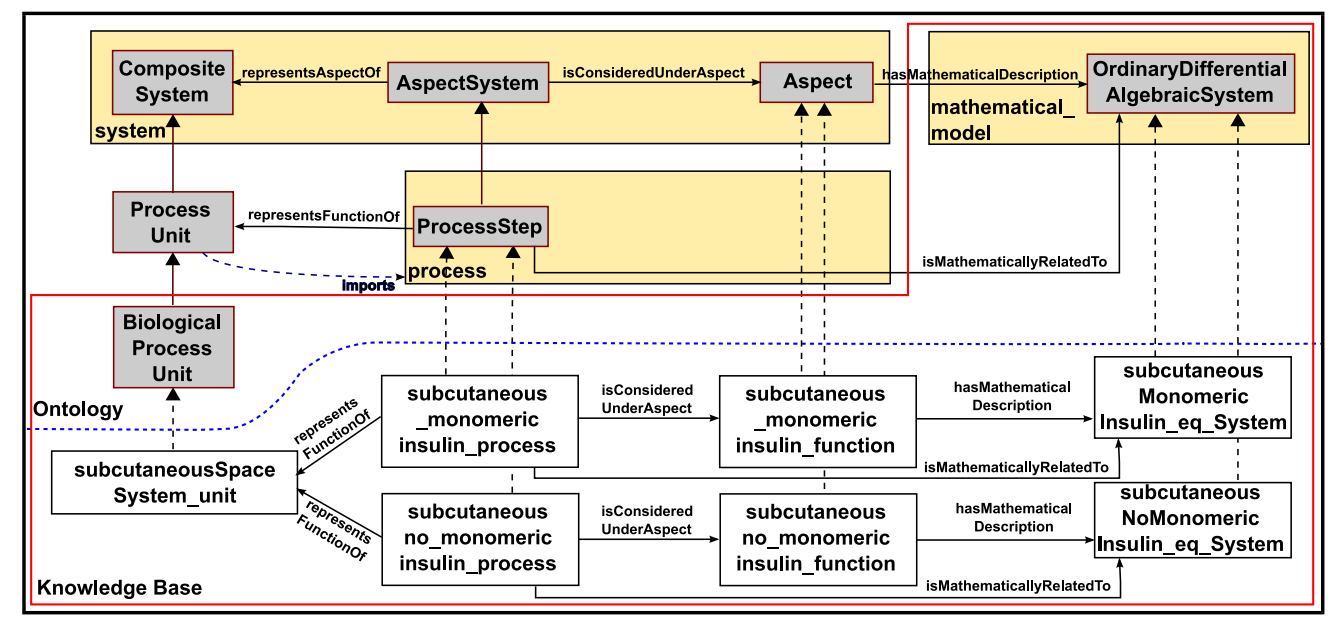

(a) Processes associated to the subcutaneous space subsystem function

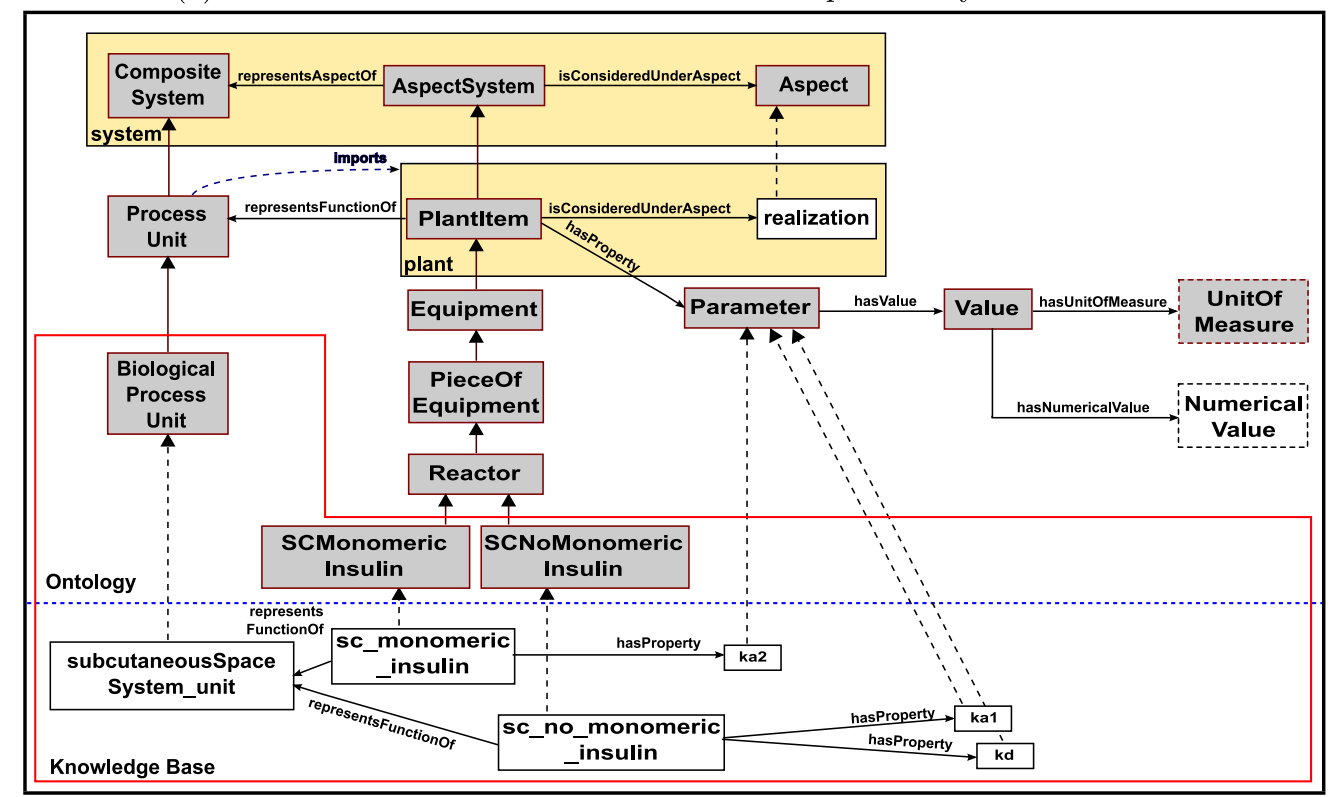

(b) Realization of the subcutaneous space subsystem
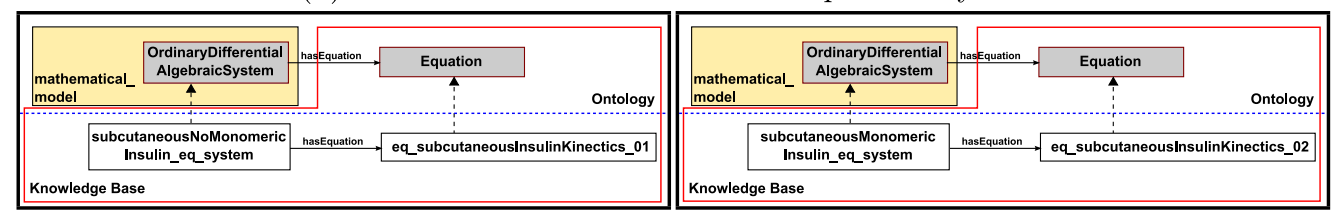

(c) Equations associated to the subcutaneous (d) Equations associated to the subcutaneous no monomeric insulin function monomeric insulin function

Fig. B.18. Subcutaneous space subsystem. 


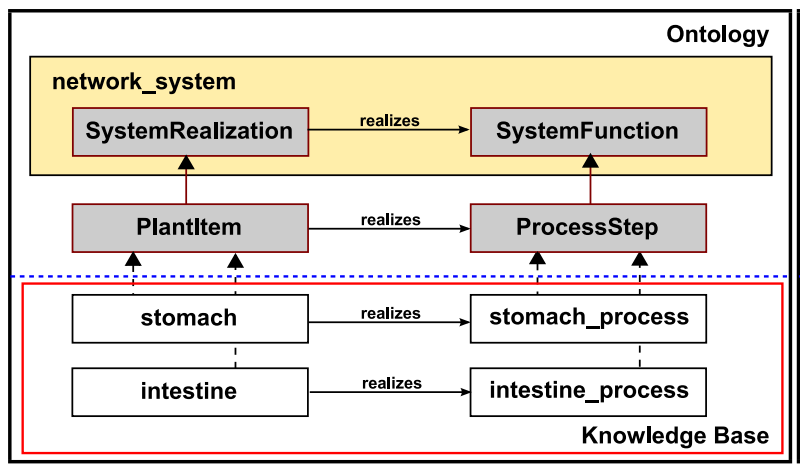

(a) Absorption subsystem realization connections

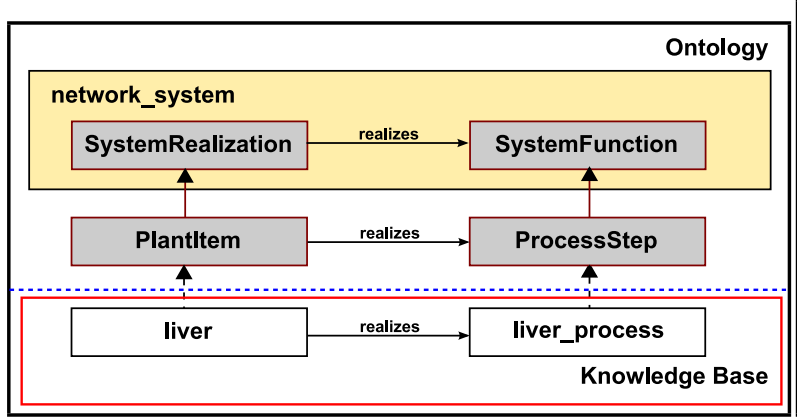

(c) Liver subsystem realization connections

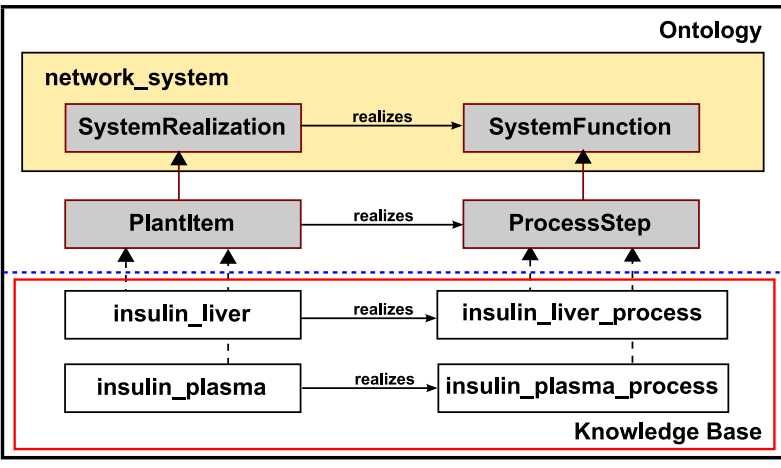

(b) Insulin subsystem realization connections

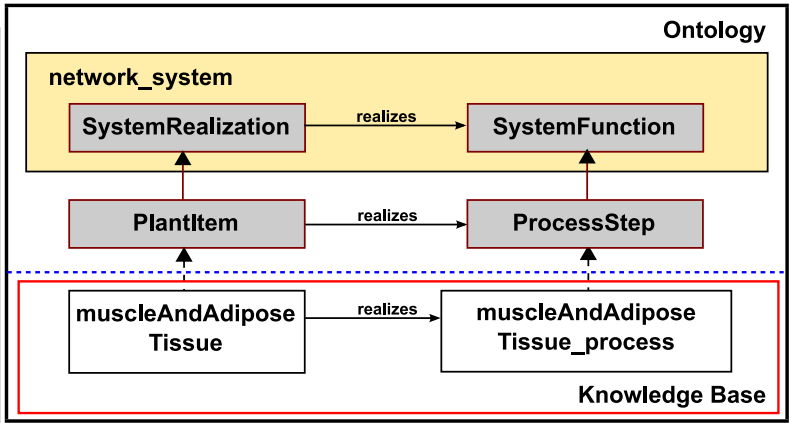

(d) Muscle and adipose tissue subsystem realization connections

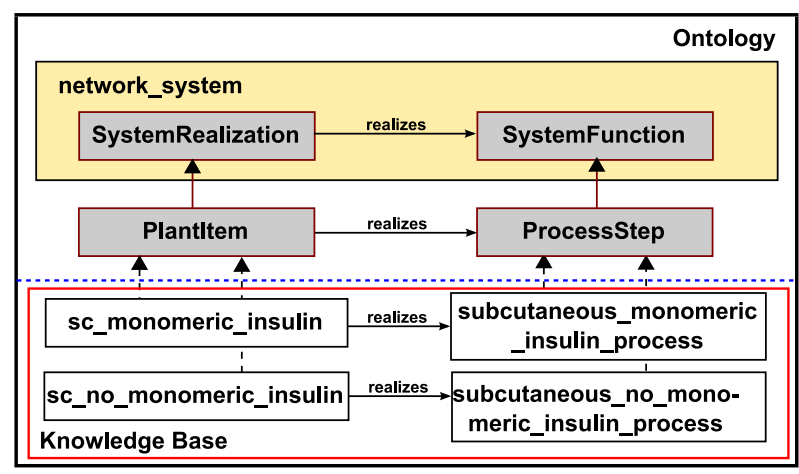

(e) Subcutaneous space subsystem realization connections

Fig. B.19. Realization connections.

\section{References}

[1] M.K. Boulos, F. Harvey, A. Roudsari, R. Bellazzi, M. Hernando, T. Deutsch, D. Cramp, E. Carson, A proposed semantic framework for diabetes education content management, customisation and delivery within the $\{\mathrm{M} 2 \mathrm{DM}\}$ project, Comput. Methods Programs Biomed. 83 (3) (2006) 188-197.

[2] J. Cantais, D. Dominguez, V. Gigante, L. Laera, V. Tamma, An example of food ontology for diabetes control, in: Proceedings of the International Semantic Web Conference 2005 workshop on Ontology Patterns for the Semantic Web, 2005.

[3] J.E. Caporale, J.F. Elgart, J.J. Gagliardino, Diabetes in argentina: cost and management of diabetes and its complications and challenges for health policy, Globalization Health 9 (54) (2013) 1-10.

[4] C. Cobelli, E. Carson, Introduction to Modeling in Physiology and Medicine, first ed., Elseiver, Burlington, 2008.

[5] P.H. Colmegna, Simulation \& Control in Type 1 Diabetes, Instituto Tecnológico de Buenos Aires, 2015 Ph.D. thesis.

[6] E. DM, S. L., Archimedes: a trial- validate model of diabetes, Diabetes Care 26 (11) (2003) 3093-3101.

[7] E. DM, S. L., Validation of the archimedes diabetes model, Diabetes Care 26 (11) (2003) 3102-3110.

[8] L. González, J.E. Caporale, J.F. Elgart, J.J. Gagliardino, The burden of diabetes in argentina, Global J. Health Sci. 7 (3) (2015) 124-133.
[9] T.R. Gruber, A translation approach to portable ontology specifications, Knowl. Acquisition 5 (2) (1993) 199-220.

[10] I.D.L. Bogle, R. Allen., T. Sumner, The role of computer aided process engineering in physiology and clinical medicine, Comput. Chem. Eng. 34 (5) (2010) 763-769.

[11] B.P. Kovatchev, M. Breton, C. Cobelli, C. Dalla Man, Method, system and computer simulation environment for testing of monitoring and control strategies in diabetes, 2008, (Patent WO/2008/157781).

[12] B.P. Kovatchev, M. Breton, C.D. Man, C. Cobelli, In silico preclinical trials: a proof of concept in closed-loop control of type 1 diabetes, J. Diabetes Sci. Technol. 3 (1) (2009) 44-55.

[13] B.P. Kovatchev, C. Cobelli, C. Dalla Man, System, method and computer simulation environment for in silico trials in prediabetes and type 2 diabetes, 2010 (Patent WO/2010/151834).

[14] M.C. Legaz-García, C. Martínez-Costa, M. Menárguez-Tortosa, J.T. Fernández-Breis, A semantic web based framework for the interoperability and exploitation of clinical models and ehr data, Knowl. Base Syst. 105 (2016) 175-189.

[15] C.D. Man, C. Cobelli, A system model of oral glucose absorption: validation on gold standard data, IEEE Trans. Biomed. Eng. 53 (12) (2006) 2472-2478.

[16] W. Marquardt, J. Morbach, A. Wiesner, A. Yang, OntoCAPE: A Re-Usable Ontology for Chemical Process Engineering, Springer Berlin Heidelberg, 2010, doi:10.1007/978-3-642-04655-1. 
[17] A. Minutolo, M. Esposito, G.D. Pietro, A fuzzy framework for encoding uncertainty in clinical decision-making, Knowl. Base Syst. 98 (2016) 95-116.

[18] J. Morbach, A. Yang, W. Marquardt, Ontocape-a large-scale ontology for chemical process engineering, Eng. Appl. Artif. Intell. 20 (2) (2007) 147-161.

[19] S. Nimmagadda, S. Nimmagadda, H. Dreher, Multidimensional data warehousing \& mining of diabetes \& food-domain ontologies for e-health, in: Industrial Informatics (INDIN), 2011 9th IEEE International Conference on, 2011, pp. 682-687.

[20] P. Rajbh, R. Gosai, R.C. Shah, P. Kc, Semantic web in medical information systems, Int. J. Adv. Eng. Technol. 5 (2012) 536-543.
[21] F. Roda, E. Musulin, An ontology-based framework to support intelligent data analysis of sensor measurements, Expert Syst. Appl. 41 (17) (2014) 7914-7926.

[22] Y. Wang, J. Gu, Z Zhou, Z. Wang, Diarrhoea outpatient visits prediction based on time series decomposition and multi-local predictor fusion, Knowl. Base Syst. 88 (2015) 12-23.

[23] W. Zhang, K. Thurow, R. Stoll, A knowledge-based telemonitoring platform for application in remote healthcare, Int. J. Comput. commun. Control 9 (2014) 644-654. 